\title{
Chiral Cyclobutane $\beta$-Amino Acid-Based Amphiphiles: Influence of cis/trans Stereochemistry on Solution Self-aggregation and Recognition
}

\author{
Alessandro Sorrenti ${ }^{1}$, Ona Illa ${ }^{1}$, Ramon Pons ${ }^{2, *}$, Rosa M. Ortuño ${ }^{1, *}$. \\ ${ }^{1}$ Departament de Química, Universitat Autònoma de Barcelona, 08193, Cerdanyola del Vallès, Barcelona, \\ Spain \\ ${ }^{2}$ Departament de Tecnologia Química i de Tensioactius, Institut de Química Avançada de Catalunya, \\ IQAC-CSIC, c/ Jordi Girona 18-26, 08034 Barcelona, Spain
}

\begin{abstract}
Novel diastereomeric anionic amphiphiles based on the rigid cyclobutane $\beta$-amino acid scaffold have been synthesized and deeply investigated with the aim of generating new functional supramoleculararchitectures on the basis of the rational design of original amphiphilic molecules and the control of their self-assembly. The main interest has been focused on the effect that cis/trans stereochemistry exerts on their molecular organization and recognition. In diluted solutions, the relative stereochemistry mainly influences the head group solvation and anionic-charge stabilization, i.e. better stabilized in the cis diastereoisomer due to intramolecular hydrogen-bonding and/or charge-dipole interactions. This provokes differences in their physicochemical behavior $\left(\mathrm{pK}_{\mathrm{a}}, c m c\right.$, conductivity) as well as in the structural parameters of the spherical micelles formed. Although both diastereoisomers form fibers that evolve with time from the spherical micelles, they display markedly different morphology and kinetics of formation. In the lyotropic liquid crystal domain, the greatest differences are observed at the highest concentrations, and can be ascribed to different hydrogen-bonding and molecular packing imposed by the stereochemical constraints.
\end{abstract}

Remarkably, the spherical micelles of the two anionic surfactants show dramatically diverse enantioselection ability for bilirubin enantiomers. In addition, both the surfactants form heteroaggregates 
with bilirubin at submicellar concentrations, but with a different expression of supramolecular chirality. This points out that the unlike relative configuration of the two surfactants influences their chiral recognition ability, as well as the fashion in which chirality is expressed at the supramolecular level by controlling the molecular organization in both micellar aggregates and surfactant/bilirubin heteroaggregates. All these differential features can be appropriate and useful for the design and development of new soft materials with predictable and tunable properties, and reveal the cyclobutane motif as a valuable scaffold for the preparation of new amphiphiles. 


\section{INTRODUCTION}

A lot of investigations are being carried out focusing in the search of new amphiphiles with original or improved properties due to their growing fields of applications. ${ }^{1}$ They are used in supramolecular chemistry because of their capability to self-organize into supramolecular nanostructures from micellar fibers to smart hydrogels, for instance., ${ }^{2,3}$ Several of them are amino acid-based amphiphiles conferring absolute chirality to these molecules. This is relevant because molecular chirality seems to be intimately associated with the growth of self-assemblies with high aspect ratios and, consequently, with the formation of hydrogels. ${ }^{4}$ It is noteworthy that when an amphiphile contains more than one stereogenic center, their relative stereochemistry strongly affects its ability to form nanofibers, their morphology and, therefore, its gelation behavior. ${ }^{5}$ Moreover, amphiphile aggregates such as micelles, liposomes, and Langmuir monolayers are useful models to investigate molecular and chiral recognition in complex noncovalent polymolecular assemblies, which is a crucial aspect in supramolecular chemistry. These features make amphiphile aggregates simple models for studying the organization and functions of biomembranes. ${ }^{6,7}$ Some investigations have used racemic mixtures of rapidly interconverting enantiomers, e.g. biphenylic derivatives and bilirubin, as markers of chirality. They showed that the expression of chirality in amphiphile self-assemblies cannot be easily correlated with the chiral information embedded in the monomer, but it depends on the organization of the whole aggregate. ${ }^{8,9}$

Amphiphiles also find application in nanotechnology and materials chemistry. For instance, conventional amphiphile aggregates such as micelles, rods and liquid crystals have been used as templates for mesoporous silica and zeolite formation. ${ }^{10}$ One of the first mesoporous silicas with inherent chirality was achieved by using chiral sodium $N$-acylalanates. ${ }^{11}$ Both silica and metal oxide nanostructures (e.g. fibers and tubes) have also been achieved by using amphiphiles as templates, with potential applications in nanotechnology, as optical devices, in drug delivery and ion sensing. ${ }^{12,13}$

Further, there is a growing interest in the application of amphiphiles in biological and medicinal chemistry. For example, peptide-based amphiphiles have been used in the preparation of materials mimicking the complex structure and properties of bone. ${ }^{14}$ In addition, conveniently functionalized 
amphiphiles produce assemblies that are good candidates for drug design ${ }^{15}$ or they are used as soft delivery systems. ${ }^{16}$ Lastly, the ability to combine bioinspired strategies to build artificial molecular devices with the possibility of imparting responsive and tunable properties has an impressive impact on nanoscience and soft-matter science. ${ }^{17}$

The chemical nature of the investigated amphiphiles is manifold, although many of them are based on natural amino acids. Besides other relevant properties (e.g. chirality, presence of ionizable groups and possibility of hydrogen bonding), they are biodegradable and biocompatible. ${ }^{18,19}$

In this context, the search for new scaffolds for amphiphile molecules, as well as the elucidation and control of the mechanisms by which the molecular information is transferred to the morphology, organization and functions of complex supramolecular architectures, are challenging research fields. The aim is to find out new molecular design rules and to master intermolecular interactions, which is crucial for the generation of novel functional nanostructures through a bottom-up strategy.

Unnatural cyclobutane $\beta$-amino acid derivatives, namely 1,2-disubstituted cyclobutanes, afford a suitable chiral frame for the preparation of diverse types of compounds. The rigidity conferred by the cyclobutane ring together with the relative cis or trans stereochemistry determines not only the preferred conformation in solution, ${ }^{20}$ but the mode of aggregation of several kinds of unnatural peptides, ${ }^{21}$ and functional organic fibers $^{22}$ among other materials. Thus, while oligomers consisting of monomers derived of cis-2aminocyclobutane-1-carboxylic acid adopt extended conformations in solution as the consequence of intra-residual hydrogen-bonding, ${ }^{23}$ the analogous trans-oligomers give rise to the formation of helices due to inter-residual hydrogen bonds. ${ }^{24}$ In both cases, secondary structures result from the formation of intramolecular hydrogen-bonds (see Chart S1 in SI). The influence of cis/trans-stereochemistry has also been manifested in the tendency of cyclobutane-containing $\beta$-dipeptides to assemble into nanoscale fibers that interact with one another (non-covalent interactions) to form solid-like networks. In this case, both experimental and computational studies suggested a different gel packing as the result of distinct molecular arrangements in the self-assembly formation process leading to helical structures corresponding to hydrogen-bonded single chains. ${ }^{25}$ 
In this work, we report on the preparation and study of new chiral cyclobutane $\beta$-amino acid-based amphiphiles (Figure 1a) accounting for the influence of cis/trans stereochemistry on their molecular organization and chiral recognition. Cis-and trans-anionic surfactants $\mathbf{1}$ were investigated regarding their aggregation behavior in water as well as their ability to form lyotropic liquid crystals. The expression of chirality in their aggregates was investigated by studying the chiral recognition of bilirubin. In all cases, a strong effect of the relative cis/trans stereochemistry was evidenced.

\section{EXPERIMENTAL SECTION}

Synthesis of $N$-lauroyl amphiphiles. The detailed synthesis and characterization of surfactants $\mathbf{1}$ and their precursors are reported in SI.

Sample Preparation. The aqueous samples for surface tension were prepared by successive dilution starting from a concentrated mother solution $(\sim 3.2 \mathrm{wt} \%)$ obtained by direct weighting of the surfactant and Millipore water $(\sim 32.1 \mathrm{mg}$ in $\sim 0.97 \mathrm{~g}$ of water). The concentration range investigated was between 0.05 and $100 \mathrm{mmol} \mathrm{Kg}^{-1}$. All measurements were performed at $25^{\circ} \mathrm{C}$ at which both surfactants $\mathbf{1}$ are totally soluble in water at the investigated concentrations, indicating that their Krafft Points are below $25^{\circ} \mathrm{C}$. Samples for $\mathrm{pH}$ and conductometric titrations were prepared by adding successive aliquots of a surfactant mother solution $\left(\sim 100 \mathrm{mmol} \mathrm{Kg}^{-1}\right)$ to a known volume of Millipore water.

Surface tension measurements. The surface tension of small volumes was measured using a home-made pendant drop tensiometer which analysis is based on the application of the Young-Laplace equation to the drop profile ${ }^{26}$ In this technique, a surfactant solution drop is created at the end of a straight cut tiny Teflon tube, which has an internal diameter of $0.8 \mathrm{~mm}$ and an external diameter of $1.58 \mathrm{~mm}$. The image of the drop is recorded using a web cam (640x480 pixels) and corrected for spherical aberration. The drop profile is then extracted from the corrected drop image after background subtraction. The droplet contour is taken at the point of maximum slope of the intensity. This contour is fitted to the Laplace-Young equation using a home-made Golden Section Search Algorithm. The input parameters of this algorithm are the reference framework, an angular correction for the vertical alignment, the radius of the droplet at 
the apex, the deformation parameter, and the interfacial tension. Care was taken to ensure a saturated humidity atmosphere to prevent evaporation. Temperature was maintained at $25.0 \pm 0.5^{\circ} \mathrm{C}$. Pure water surface tension measurements were found in a range of $70 \pm 2.0 \mathrm{mNm}^{-1}$. Further checking of the setup with ethanol found values of $21.2 \pm 1.0 \mathrm{mNm}^{-1}$, both standards agreeing with published values of $72.0 \mathrm{mNm}^{-1}$ and $21.9 \mathrm{mNm}^{-1}$ at $30{ }^{\circ} \mathrm{C}$

Surface tension was followed as a function of time till equilibrium was reached (not appreciable variation of $\gamma$ ) which happened within 4-5 h from sample preparation.

The surface area per surfactant molecule at the interface was calculated from the slope of surface tension versus the logarithm of surfactant concentration plot, just before the cmc, using the Gibbs adsorption isotherm (see main text):

$$
A_{m}=-\left(10^{18} n R T / N_{A}\right) \cdot(d \gamma / d \operatorname{lnC})^{-1}
$$

where $\gamma$ is the surface tension in $\mathrm{Nm}^{-1}, A_{m}$ is the minimum surface area expressed in $\mathrm{nm}^{2}, \mathrm{C}$ is the surfactant concentration, $R=8.314 \mathrm{~J} \mathrm{~mol}^{-1} \mathrm{~K}^{-1}, N_{A}$ is the Avogadro's number, $\mathrm{T}$ is the absolute temperature, and $n$ the effective number of dissociated species per molecule $(\mathrm{n}=2$ for the anionic surfactant without added salt).

Conductimetry. The measurements were performed with a Thermo Orion conductimeter model 150 coupled with an Orion 011020 parallel-plate cell (cell constant $0.094 \mathrm{~cm}^{-1}$ ). The cell was calibrated with standard sodium chloride solutions. Measurements were done at increasing surfactant concentration to minimize errors from possible contamination of the electrode. The measurements were performed at $25^{\circ} \mathrm{C}$ and under nitrogen atmosphere to avoid $\mathrm{CO}_{2}$ solubilization. The $c m c$ was determined from the concentration at the intersection of the linear portions of the conductivity versus concentration plot.

pH measurements. The $\mathrm{pH}$ measurements were performed with a Thermo Orion model 720A pH-meter at increasing surfactant concentration using an Orion 8103SC Ross Semimicro combination electrode. The acid-base equilibrium was modelled by the Equation 2 for a weak base, assuming dilute ideal 
behaviour and complete salt dissociation. Equation 2 was obtained taking into account the equilibrium constant definition, mass and charge balances and ionic water product (see SI for the details).

$\mathrm{C}_{\mathrm{b}}=\left(K_{a}+\left[\mathrm{H}^{+}\right]\right) \cdot\left(K_{w} /\left[\mathrm{H}^{+}\right]^{2}-1\right)$

The $\mathrm{pK}_{\mathrm{a}}$ values both below the $c m c$ (surfactant as monomer) and above the $c m c$ (apparent $\mathrm{pK}_{\mathrm{a}}$ ) were evaluated by using Equation 2.

Spectroscopic measurements. Circular Dichroism (CD) spectra were recorded on a Jasco J-715 spectropolarimeter, using quartz cells with different light path $(0.01-1 \mathrm{~cm})$, depending on sample concentration, in order to keep the photomultiplier voltage (HT) below a $600 \mathrm{~V}$ on the entire wavelength range. UV-Vis spectra were recorded on a HP/Agilent 8453 Spectrophotometer.

Small-Angle X-ray Scattering (SAXS). SAXS measurements were carried out using a S3-MICRO (Hecus X-ray systems GMBH Graz, Austria) coupled to a GENIX-Fox 3D X-ray source (Xenocs, Grenoble), which provides a detector focussed x-ray beam with $\lambda=0.1542 \mathrm{~nm} \mathrm{Cu} \mathrm{K}$-line with more than 97\% purity and less than $0.3 \% \mathrm{~K}_{\beta}$. Transmitted scattering was detected using a PSD 50 Hecus. Temperature was controlled by means of a Peltier TCCS-3 Hecus. The samples were inserted in a flowthrough glass capillary $1 \mathrm{~mm}$ diameter with $10 \mu \mathrm{m}$ wall thickness. The SAXS scattering curves are shown as a function of the scattering vector modulus:

$$
q=(4 \pi / \lambda) \cdot \sin (\theta / 2)
$$

where, $\theta$ is the scattering angle. The $\mathrm{q}$ values with this setup ranged from $0.08 \mathrm{~nm}^{-1}$ to $6.0 \mathrm{~nm}^{-1}$. The system scattering vector was calibrated by measuring a standard silver behenate sample. Because of the use of a detector focussed small beam ( $300 \times 400 \mu \mathrm{m}$ full width at half maximum) the scattering curves are mainly smeared by the detector width. This smearing mainly produces a widening of the peaks without noticeable effect on the peak position in the small angle regime. The scattering curves for liquid samples have been background subtracted and put in absolute scale by comparison with a water sample scattering. ${ }^{27,28}$ The instrumentally smeared experimental SAXS curves were fitted to numerically smeared models for beam size and detector width effects. A least squares routine based on the Levenberg- 
Marquardt scheme was used. Discoidal and cylindrical form factors were fitted using core-shell models. ${ }^{29,30}$ Hexagonal liquid crystal was fitted using cylindrical core-shell form factor ${ }^{31}$ and structure factor taken from Ref. 32.

Optical and Electron Microscopy. Polarizing optical micrographs were obtained with a Zeiss microscope. The images were taken with a Canon PowerShot S90 digital camera opportunely adapted. TEM images were recorded with a JEOL JEM 2010 operating at 200kV and a JEOL JEM 1400 operating at $120 \mathrm{kV}$. A drop of the surfactant solution $(5 \mu \mathrm{l})$ was placed on a carbon coated copper grid and after 10 s the excess of water was blotted off with filter paper. The images were recorded without staining.

\section{RESULTS AND DISCUSSION}

Synthesis of the cyclobutane-based amphiphiles 1. Compounds cis- and trans-1 were prepared following an analogous route (Figure 1b) starting from the appropriate $N$-Boc protected cyclobutane $\beta$ amino esters cis- and trans-2, respectively, which in turn were prepared in enantiomerically pure form as previously reported ${ }^{25}$ Direct attempts to condensate the free amino acids with lauroyl chloride did not work. As a matter of fact, the typical acylation conditions used for the natural amino acids (often implying the use of an aqueous medium) were not applicable here, due to the instability of the cyclobutane free amino acids, which easily undergo ring opening in such conditions. ${ }^{25}$ 
(a)

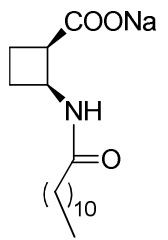

cis-1

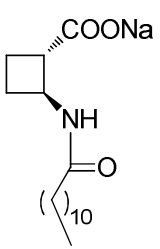

trans-1

(b)

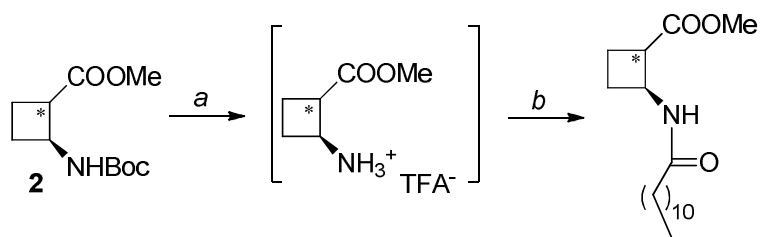

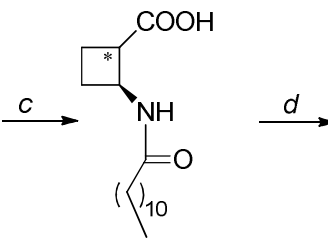

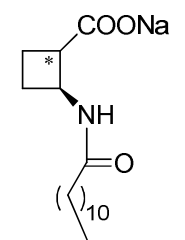

3

Figure 1. (a) Structure of anionic surfactants 1. (b) Synthesis of cis- and trans-1, reagents and conditions; $a$ : TFA, $\mathrm{Et}_{3} \mathrm{SiH}_{\text {, }}$ $\mathrm{CH}_{2} \mathrm{Cl}_{2}, 0{ }^{\circ} \mathrm{C}, 3 \mathrm{~h}$, quantitative; $b: \mathrm{C}_{11} \mathrm{H}_{23} \mathrm{COCl}, \mathrm{Et}_{3} \mathrm{~N}, \mathrm{CH}_{2} \mathrm{Cl}_{2}, 0{ }^{\circ} \mathrm{C}, 5$ h, $77 \% ; c: 0.25 \mathrm{M} \mathrm{NaOH}$, r.t., 5 h, $95 \%$; $\mathrm{NaHCO}_{3}, \mathrm{THF}-\mathrm{H}_{2} \mathrm{O}$, ultrasounds, $4 \mathrm{~h}, 80 \%$.

For that reason, we decided to perform the $N$-acylation on the corresponding amino esters. Thus, the synthetic sequence starts with the selective removal of the $N$-Boc carbamate by treatment of fully protected amino acid 2 with trifluoroacetic acid in the presence of $\mathrm{Et}_{3} \mathrm{SiH}$. The resulting trifluoroacetate salt, without further purification, was submitted to reaction with lauroyl chloride, in the presence of triethylamine, to afford ester $\mathbf{3}$ in $77 \%$ yield over the two steps. Saponification of the methyl ester under mild conditions $(0.25 \mathrm{M} \mathrm{NaOH})$ led to the formation of acids 4 in almost quantitative yield. Finally, treatment of 4 with $\mathrm{NaHCO}_{3}$ in tetrahydrofurane-water under ultrasounds activation led to the preparation of salts 1 in $80 \%$ yield. IR spectra of the sodium salts showed the disappearance of the $\mathrm{C}=\mathrm{O}$ stretching around $1700 \mathrm{~cm}^{-1}$ and the appearance of strong bands near 1600 and $1400 \mathrm{~cm}^{-1}$, corresponding to the antisymmetric and symmetric stretching of the carboxylate group. ${ }^{33}$ It was possible to easily verify the complete salification (no acid remains) by comparing the ${ }^{1} \mathrm{H}$ NMR spectrum of the acid and the salt in 
$\mathrm{CD}_{3} \mathrm{OD}$. In fact, the corresponding resonance for $\mathrm{H}_{1}$ moves somewhat from lower to higher fields with respect to the residual proton of the solvent upon salification.

Aggregation behavior in diluted water solution. In order to investigate the aggregation behavior of the two diastereomeric anionic surfactants cis- and trans-1 in diluted water solution, their critical micellar concentration $(\mathrm{cmc})$ was determined by surface tension and conductivity measurements. Moreover, their acid-base character was investigated at different surfactant concentrations.

The surface tension $(\gamma)$ of the two anionic surfactants decreases progressively upon increasing concentration (Figure 2a) till a break above which it almost levels to a constant value $\left(\sim 33 \mathrm{mN} \cdot \mathrm{m}^{-1}\right)$. Because of the small minimum found for $\mathbf{c i s} \mathbf{- 1}$, the sample was purified further by two recrystallizations and using the foam purification method. ${ }^{34}$ The minimum could not be reduced even after 24-hour foaming, which likely suggests that its origin is not due to the presence of hydrophobic impurities. The break corresponds to the $c m c$ that was determined as the intersection of two fitting straight lines in the plot of $\gamma$ versus $\log \mathrm{c}$. From the slope just before the break, the minimum area per molecule $\left(A_{m}\right)$ was calculated according to the Gibbs adsorption isotherm using 2 as the number of adsorbing species (see the Experimental Section). The $c m c$ and $A_{m}$ values for the two surfactants are reported in Table 1. The area per molecule, as obtained from the Gibbs isotherm, seems rather large; this could be due to the overestimation in the number of adsorbed species used in the equation. In fact, part of the surfactant may lose the ionic character because of changes in the apparent $\mathrm{pK}_{\mathrm{a}}$ induced by aggregation (see below). Also we should bear in mind other possible sources of error associated with the application of Gibbs isotherm to determine the adsorption of surfactants at interfaces. ${ }^{35}$

Table 1. Surface and acid-base properties of surfactants cis- and trans-1 in water at $25^{\circ} \mathrm{C}$

\begin{tabular}{|l|ll|ll|l|}
\hline \multirow{2}{*}{ Compound } & \multicolumn{2}{|l|}{$c m c\left(\mathrm{mmol} \cdot \mathrm{Kg}^{-1}\right)$} & $\mathrm{pK}_{\mathrm{a}}$ & & $A_{m}\left(\mathrm{~nm}^{2}\right)^{\mathrm{a}}$ \\
\cline { 2 - 6 } & surface tension & conductivity & {$[\mathbf{1}]<c m c$} & {$[\mathbf{1}]>c m c$} & \\
\hline cis-1 & $8 \pm 2$ & $12.7 \pm 2.5$ & $4.12 \pm 0.05$ & $5.59 \pm 0.05$ & $1.5 \pm 0.1$ \\
trans-1 & $5 \pm 2$ & $8.5 \pm 1.7$ & $4.35 \pm 0.05$ & $5.72 \pm 0.05$ & $1.3 \pm 0.1$
\end{tabular}

${ }^{a}$ Determined from surface tension parameters 
Figure $2 \mathrm{~b}$ shows the conductivity as a function of the concentration of aqueous solutions of the two anionic surfactants. The conductivity increases with increasing concentration and a clear inflection is observed corresponding to the $c m c$ value. As expected, above the $c m c$ the slope is lower than below, being the large and partially charged micelles worse charge carriers when compared with the surfactant monomers. Note that the $c m c$ values obtained by conductimetry are larger than those obtained from surface tension measurements (Table 1). The differences of $c m c$ measured for the two diastereomeric surfactants are small but consistent with the different techniques used, which in turn depend on distinct physical phenomena. ${ }^{36}$ When compared to literature, both values agree with those of classical ionic surfactants with a dodecyl hydrophobic chain. ${ }^{37}$

a)

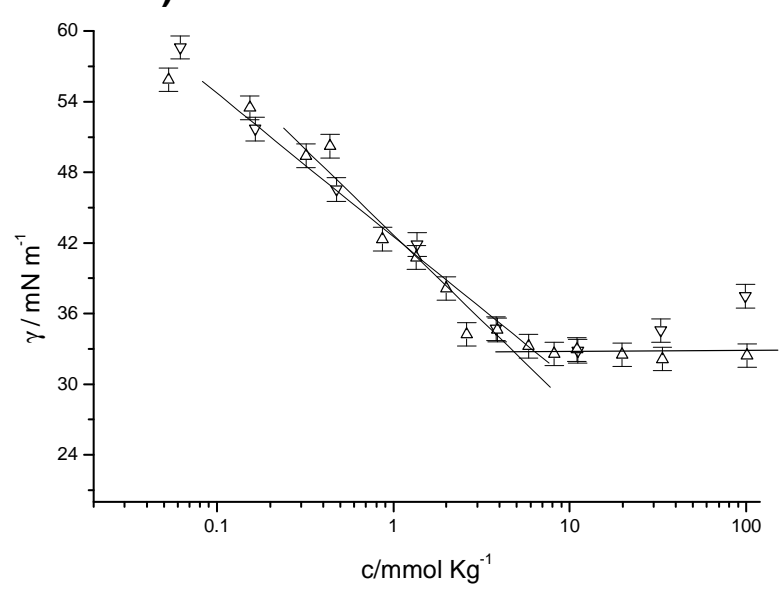

b)

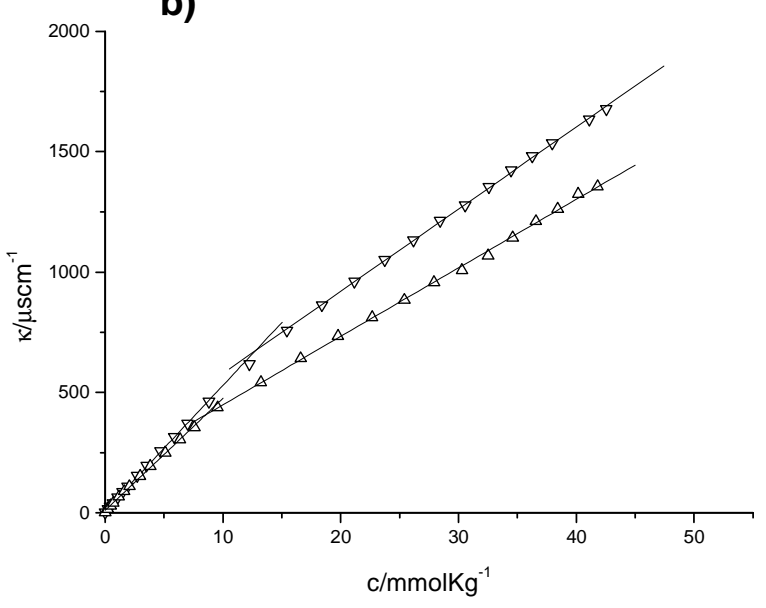

Figure 2. Plot of surface tension (a) and conductivity (b) as a function of surfactant concentration for cis-1 (down triangles) and trans-1 (up triangles) in water at $25^{\circ} \mathrm{C}$.

The $\mathrm{pH}$ was measured as a function of surfactant concentration at $25^{\circ} \mathrm{C}$ as shown in Figure 3 . Below the $c m c$, when surfactants are in the monomeric form, the acid-base behavior was modeled with that of a weak base according to Equation 2 in the Experimental Section, from which the $\mathrm{pK}_{\mathrm{a}}$ of the conjugated acid was obtained (Table 1). Interestingly, monomer cis-1 behaves as a slightly weaker base than monomer trans-1. However, an increase in basicity (increase of the apparent $\mathrm{pK}_{\mathrm{a}}$ ) is observed for both surfactants around the $c m c$. The change appears at lower concentration for trans-1 than for cis-1 (Figure 3) as expected from the $c m c$ values obtained from conductivity and surface tension. This increase in basicity is reasonable because more and more protons are captured by the carboxylate anion in order to 
shield partially the electrostatic repulsion (high density of negative charge) between the anionic surfactant heads on the surface of the aggregates.

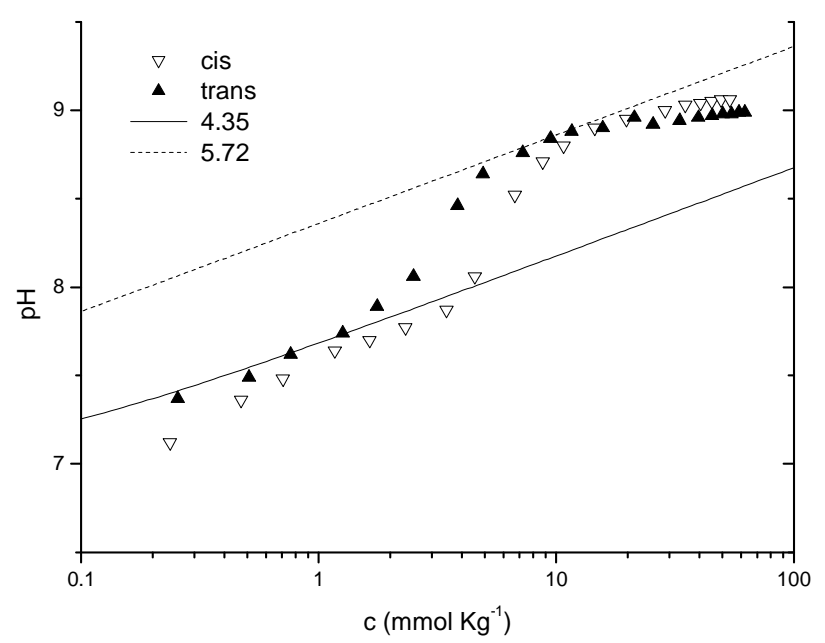

Figure 3. Plot of $\mathrm{pH}$ as a function of surfactant concentration for cis-1 (down triangles) and trans-1 (full up triangles) in water at $25^{\circ} \mathrm{C}$. The curves represent the variation of $\mathrm{pH}$ as a function of concentration of weak bases with $\mathrm{pK}_{\mathrm{a}}$ values of 4.35 (full line) and 5.72 (dashed line) calculated according to Equation 2.

This effect becomes less important as the ionic strength increases. In Figure 3 one can observe that below the $c m c$ the $\mathrm{pH}$ of trans-1 solutions changes with surfactant concentration following reasonably Equation 2 with $\mathrm{pK}_{\mathrm{a}}=4.35 \pm 0.05$. The basicity increases continuously up to $\mathrm{pK}_{\mathrm{a}}=5.72 \pm 0.05$ around $6-8 \mathrm{mmol} \cdot \mathrm{Kg}^{-}$ 1. The behavior of $\boldsymbol{c i s} \mathbf{- 1}$ is analogous to that of trans-1 with slightly lower values of $\mathrm{pK}_{\mathrm{a}}(4.12 \pm 0.05$ and $5.59 \pm 0.05$, respectively). Similar conclusions can be drawn when considering the apparent $\mathrm{pK}_{\mathrm{a}}$ plotted as a function of surfactant concentration (Figure $\mathrm{S} 1$ in the $\mathrm{SI}$ ). While below the $c m c$ the $\mathrm{pK}_{\mathrm{a}}$ is constant, above the $c m c$ the $\mathrm{pK}_{\mathrm{a}}$ trend changes and progressively diminishes again, probably due to the increase in ionic strength. The differences in $\mathrm{pK}_{\mathrm{a}}$ between the two diastereoisomers (behavior of cis-1 as a weaker base than trans-1) can be attributed to the different stabilization of the acid-base species by hydrogen bonding and/or charge dipole interactions. Thus, the anionic form of $\mathbf{c i s} \mathbf{- 1}$ has a higher stability compared with that of trans-1. This different stability agrees with the negative charge of the carboxylate group being partially stabilized by intramolecular hydrogen bonding. The difference is also maintained around the $c m c$, but cis-1 shows a smaller net charge per molecule than trans-1 well above the $c m c$ (i.e. stronger 
basicity). This would seem contrary to what is shown in Figure 2 for the conductivity. However, while the pH measurements concern only the acid-base equilibrium, the conductivity reflects the global conductivity of species present in solution including protons and hydroxide anions as well as their counterions. Taking into account the $\mathrm{pH}$ measurements, the expected conductivity can be calculated below the $\mathrm{cmc}$ using the ionic equivalent conductivity and experimental values of surfactant concentration and $\mathrm{pH}$. Above the $\mathrm{cmc}$ the counterion binding can be estimated assuming that the micellar conductivity is small compared to the others. The results imply that the counterion binding is slightly stronger for the trans-1 micelles than for those of cis-1 (0.42 compared to 0.31$)$.

To sum up, the higher $c m c$ and area per molecule at the $c m c$ of $c$ is-1, as well as its weaker basicity as a monomer, are both consistent with a more stabilized ionic charge. This suggests a stronger hydrophilic character of cis-1 when compared with trans-1, probably due to a different solvation pattern of the polar heads imposed by the different relative stereochemistry, which also influences their $\mathrm{pK}_{\mathrm{a}}$ shifts upon micellization.

Small-angle X-Ray scattering (SAXS) studies. SAXS profiles of 3.3 wt \% solutions of the surfactants diluted in water are shown in Figure 4, where the best fits are also shown. The scattering patterns of both surfactants show a band at intermediate $q$ values, which are lower for trans-1. The behavior of trans-1 samples and freshly prepared cis-1 samples is similar at lower $q$ values (Figure S2 in the SI), while there is a clear increase for 24 hours aged cis-1 samples (Figure 4, and S3 in the SI). The best fit was obtained with a core-shell spherical micelles model in the case of trans-1 and freshly prepared cis-1 samples, while, for aged cis-1, improved fits (especially at small $q$ ) were obtained by using a core-shell cylindrical model. From the models, two main parameters were obtained, namely the micellar core and the micellar radii (Table 2). 


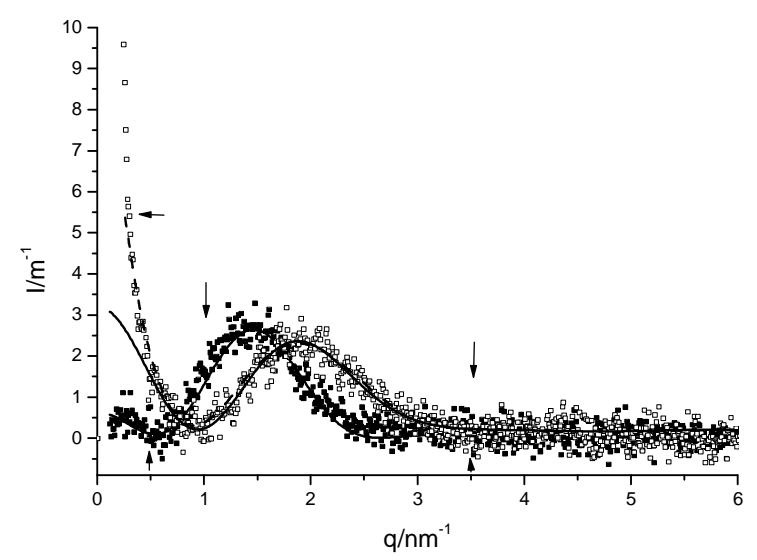

Figure 4. Intensity as a function of scattering vector modulus for trans-1 (full symbols) and 24 h aged cis-1 (open symbols). The full lines show the best fit of core-shell spheres. The up arrows show the fitting range for trans-1 while the down arrows show the fitting range for cis-1. The dashed line corresponds to the core-shell cylinder fit for cis-1; in this case the left pointing arrow marks the left limit of the fitting range. The error bars are not shown for clarity and correspond roughly to the dispersion of the experimental points.

The micellar radius is bigger for trans-1 than for cis-1. These differences also correspond to different aggregation numbers (51 for trans $\mathbf{- 1}$ compared to 29 for the fresh cis-1 and bigger for the cylinders). The corresponding area per molecule is very similar for the spherical micelles (Table 2) and the evolution to cylinders implies only a small change (a reduction from the $0.65 \mathrm{~nm}^{2}$ to $0.54 \mathrm{~nm}^{2}$ ).

Table 2. Micellar radius, micellar core radius, cylinder radius, aggregation number and area per molecule $\left(A_{m}\right)$ deduced from SAXS studies on cis- and trans-1

\begin{tabular}{|c|c|c|c|c|}
\hline Compound & $\begin{array}{l}\text { Micellar } \text { radius }^{\mathrm{a}} \\
(\mathrm{nm})\end{array}$ & $\begin{array}{l}\text { Micellar core } \text { radius }^{\mathrm{a}} \\
(\mathrm{nm})\end{array}$ & $\begin{array}{l}\text { Aggregation } \\
\text { number }\end{array}$ & $\begin{array}{l}A_{m} \\
\left(\mathrm{~nm}^{2}\right)\end{array}$ \\
\hline trans-1 & $3.1 \pm 0.3$ & $1.6 \pm 0.1$ & $51 \pm 10$ & $0.60 \pm 0.06$ \\
\hline \multirow{2}{*}{ cis-1 } & $2.1 \pm 0.2$ & $1.5 \pm 0.1$ & $29 \pm 6$ & $0.65 \pm 0.10$ \\
\hline & $1.9 \pm 0.2$ & $1.2 \pm 0.1$ & - & $0.54 \pm 0.07$ \\
\hline
\end{tabular}

${ }^{a}$ According to a core-shell spherical micelles model, or cylinder radius determined according to a core-shell cylindrical model for the aged cis-1 samples.

The diversity in the $A_{m}$ values calculated from the surface tension slope (see Table 1) or from SAXS can arise from overestimation of the number of adsorbed species in the Gibbs isotherm, while for the SAXS value it depends on the exact definition of the polar-apolar dividing interface, here taken at the $\mathrm{CO}-\mathrm{CH}_{2}$ 
bond. However, as stated before, one should be cautious concerning the values of area per molecule obtained by application of Gibbs isotherm to surface tension measurements. ${ }^{35}$

The differences in the aggregates formed by the two diastereoisomers can be explained by the differences in packing of the polar heads and the interplay between the $\mathrm{pK}_{\mathrm{a}}$ values and $\mathrm{pK}_{\mathrm{a}}$ shift, which can be also affected by intermolecular hydrogen bonding constraints (see below). However, it should be noted that the amount of the anionic deprotonated form dominates, with the contribution of the protonated species well below one percent, at the concentrations considered. Thus, the contribution of these last species to the area per molecule is limited. This induces to think that the strongest effect should be due to the packing and the charge repulsion. It is noteworthy that, in the case of trans-1 and fresh cis-1 the hydrophobic core radius corresponds to fully extended $C_{11}$-hydrophobic chains while the hydrophobic radius of the cylinders at longer times corresponds to $80 \%$ of the sphere radius. This implies a more relaxed state for the hydrophobic chain and could be one of the driving forces for this transformation. Moreover, the fitting of cylinders extends the fitting range to smaller $q$ values but fails to cover the whole range. The steep increase at the smaller $q$ values has to be interpreted as corresponding to aggregation processes, such as the formation of fibrils from cylinders (see below). Slowly growing cylindrical structures have also been evidenced before, for example in gemini arginine surfactants ${ }^{28}$ and are thought to be the origin of gelation by small molecules. ${ }^{38}$

Formation of fibrous assemblies. Both surfactants cis- and trans-1 dissolve easily in water at the investigated concentrations, up to the liquid crystals domain (30 wt \%), giving clear homogenous solutions at $25^{\circ} \mathrm{C}$ without heating or vortex agitation. However, visible fibrous aggregates appear in the micellar samples of cis-1 after almost $24 \mathrm{~h}$ incubation and remain suspended in solution for months. Fibers can grow up to many millimeters length, and upon strong shaking they break into 2-3 $\mathrm{mm}$ fragments but do not dissolve at all. In one case, the insoluble fibrous material from the solution could be separated, using a small hook, as a skein. After freeze drying, the resultant solid was analyzed by NMR, IR, and ESI(-) MS, which confirmed that it is constituted by the anionic surfactant. This ruled out the possibility that the insoluble fibers may be formed by some degradation product or the insoluble acid cis -4 
deriving from the partial carboxylate protonation. On the other hand, in the case of surfactant trans-1 the appearance of visible insoluble fibers is observed only after 2-3 weeks from sample preparation. Furthermore, their morphology is strikingly different from cis-1 aggregates as evidenced both by optical and transmission electron microscopy (see below).

The formation of insoluble fibrous-like aggregates from vesicular solutions of anionic $N$-acylamino acid surfactants has been previously observed upon acidification at $\mathrm{pH}$ 5-6 (i.e. protonation of carboxylate group) and/or temperature decrease, and their morphology including helical aggregates was found to depend upon the head group structure (type of amino acid). ${ }^{39,40}$ The formation of hydrogen bonds and of cooperative hydrogen-bond chains between secondary amide groups in the heads of neighboring molecules have been invoked as important requirements for the formation of a variety of organized supramolecular assemblies in amphiphiles, including fibers, ribbons, helices and tubes. ${ }^{2,41}$ In the case of surfactants $\mathbf{1}$, it is remarkable the spontaneous formation of fibers upon aging from a perfectly homogeneous micellar solution, without any change of experimental conditions, since micelles are wellknown equilibrium systems that formed spontaneously by dissolving the surfactants. The hypothesis is that after the fast dissolution and formation of spherical micelles driven mainly by non-directional entropic and energetic contributions, a slower reorganization of surfactant molecules at the aggregate surface may occur, triggered by molecular recognition processes and consequent formation of hydrogenbond chains. This would correspond to slow 2D crystallization at the aggregate surface in which stereochemical constraints play a crucial role in determining the organization. ${ }^{41}$ Note that the binding forces between the head groups in the fiber can compensate (balance) the electrostatic repulsion forces inducing the formation of aggregates with lower curvature such as disk micelles and rods and eventually long fibers. This would also explain the increase of SAXS intensity at small $q$ observed with time in cis-1 samples, which is sterically more constrained than the diastereomer trans-1. To validate this hypothesis, TEM experiments were conducted with both freshly prepared $1 \%$ micellar solutions of the two surfactants as well as on aged samples. Whereas in fresh samples there was no evidence of any type of structured surfactant aggregate (2-3 nm micelles are hardly visible by TEM and broke into an amorphous organic 
layer upon drying), nice fibrous aggregates were observed in aged samples of both surfactants as shown in Figure 5 for unstained samples.
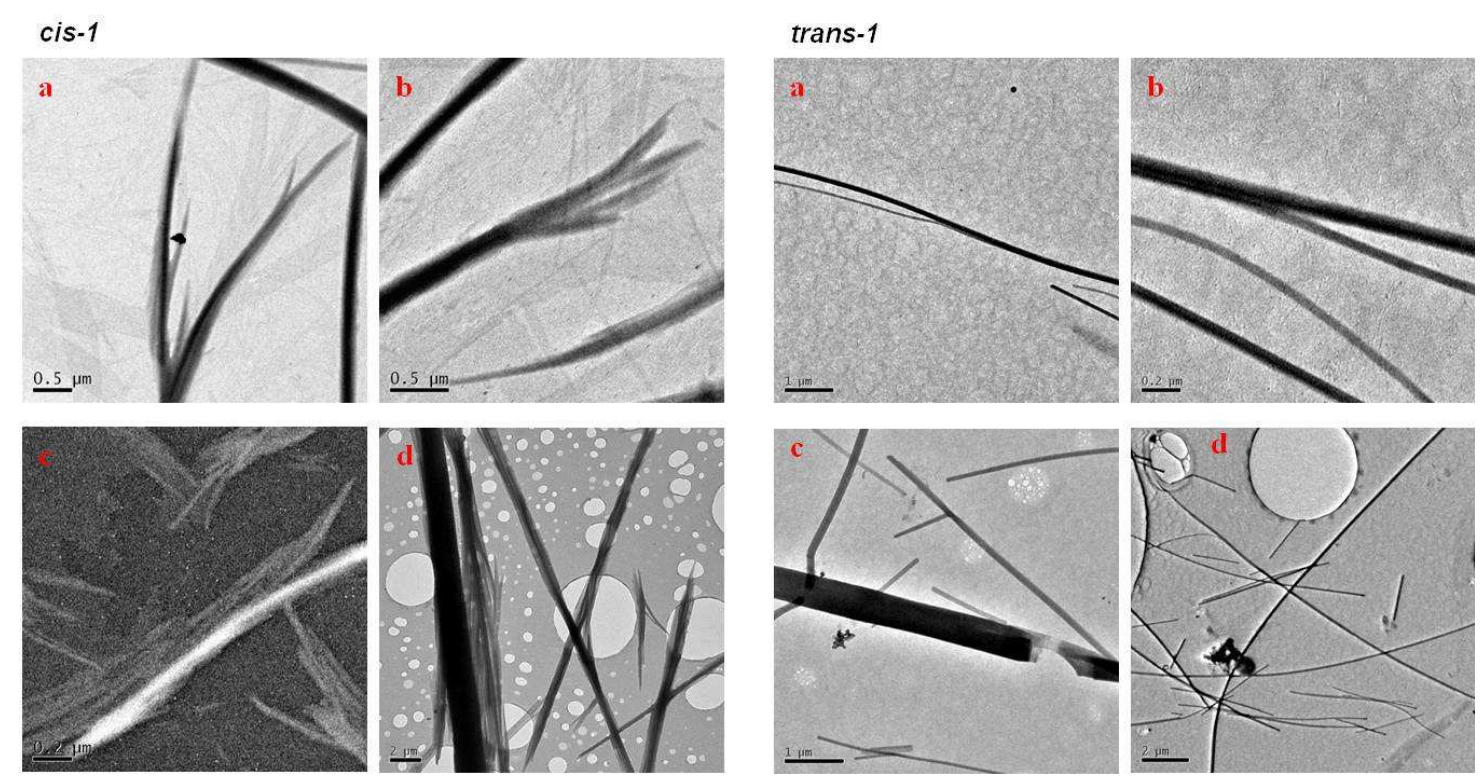

Figure 5. Electron micrographs from 1\% water solutions of $\boldsymbol{c i s}$-1 (left side): after $24 \mathrm{~h}$ incubation (a-c) and 3 weeks ageing (d), and of trans-1 (right side) after 3 weeks ageing (a-d). All images correspond to unstained samples deposited on a carboncoated copper grid.

The cis-1 fibers (left side) are ramified, have different diameters and lengths (which increase with ageing) and feature a peculiar irregular scaly surface, in which the scales are actually formed by sheets or bundles of thinner fibers, likely corresponding to the micellar rods (see Figures S4-S5 in the SI for more TEM and SEM images). This morphology is compatible with the steep increase at the smaller $q$ values observed in the scattering curves of aged cis-1 samples (Figure 4). On the other hand, trans-1 fibers are cylindrical in shape and show much more defined contours with regular ends and smooth surface, though they can have different diameters. Fibers with thicker diameters appear to be more rigid than cis-1 fibers; however, the thinner ones are still flexible enough to curve. The cylindrical aggregates can burst in a string thus revealing that they are made by bundles or ribbons of regular filaments in strong contact through side-byside interactions.

Interestingly, analogous morphological differences between fibrous aggregates of surfactants cis- and trans-1 were also observed at the greater length scale of the insoluble fibers as shown by optical 
microscopy (Figure S6 in the SI). In addition, optical micrographs show that cis-1 insoluble fibers are extremely long and flexible forming in some cases tangles, whereas trans-1 insoluble fibers are shorter and more rigid with a rod-shaped morphology.

A detailed investigation of the kinetics of fiber formation is out the scope of this article, but it should be stressed that the faster evolution of cis-1 micelles to fibers, i.e. greater tendency to evolve to lower curvature species, might depend on the smaller electrostatic repulsion due to the intermolecular stabilization of the carboxylate charge.

To sum up, the differences in the physicochemical behavior of the investigated surfactants in diluted water solution (micellar solutions) should be mainly ascribed to differences in the head group solvation and charge stabilization induced by the different stereochemistry. In turn, these features affect differently the hydrophilic/hydrophobic balance of the two surfactants. This is reasonable if we take into account that micelles are loose dynamic structures in which the stereochemical constraints hardly affect their packing and organization. However, such constraints seem to come into play in the evolution of micelles to fibrous aggregates driven by intermolecular hydrogen bonding because they influence the recognition processes at the bases of self-organization, and the possibility to form stable hydrogen-bonded networks at the aggregate surface. This stereochemical demand is further amplified by the presence of the cyclobutane moiety that restrains the amide and the carboxylate groups at fixed relative orientations.

With the aim of investigating how the relative cis/trans stereochemistry affects molecular organization in more "condensed" aggregation states, the lyotropic liquid crystalline behavior of the two anionic surfactants cis- and trans-1 in water was studied by SAXS and polarized optical microscopy.

\section{Formation of lyotropic liquid crystals from anionic surfactants cis- and trans-1.}

The SAXS profiles of cis-1 mixtures with water at increasing surfactant concentrations and of the pure solid samples are shown in Figure S7 in SI. The fitting of a hexagonal structure for the samples at the lower concentrations is reasonable in view of the diluted samples characterization. Between $30 \%$ and $45 \%$ the only significant difference in the parameters of the fit, apart from the inter cylinder distance (marked 
by the $q$ value of the first peak), corresponds to the increase of order in the sample induced by the increase of concentration (see Table 3 for the specific values). The $60 \%$ sample, however, does not follow the pattern expected for a hexagonal structure, that is, a $1,3^{1 / 2}, 4^{1 / 2}, 7^{1 / 2}, 9^{1 / 2} \ldots$ peaks position sequence. Instead, the sequence is $6^{1 / 2}, 8^{1 / 2}, 14^{1 / 2}, 33^{1 / 2}, 54^{1 / 2} \ldots$, in which the first three positions agree with a bicontinuous Ia3d cubic phase. ${ }^{42}$ This corresponds to a body-centered unit cell corresponding to the gyroid infinite minimal periodic surface which is formed by rods. This isotropic cubic structure has also been evidenced by polarizing optical microscopy as a nonbirefringent fringe in a solvent penetration experiment as shown in the photomicrograph of Figure S7 (right) in SI. Because of the sequence of first maxima in the scattering curves, the structure of this phase seems to correspond to the oil-in-water type. The fitting of the combined Ia3d structure factor with nearly the same core-shell cylinder form factor obtained for the $45 \%$ water content sample seems also to favor this view. Although the fitting is far from being perfect, the main features of the Ia3d liquid crystal structure are captured. The differences may arise from the incipient formation of a more concentrated phase.

Table 3. Structural parameters of liquid crystals mesophases of surfactants $c i s$ - and $\operatorname{trans}-1$ at $25^{\circ} \mathbf{C}$

\begin{tabular}{llll|ll|}
\hline \multirow{2}{*}{ Surfactant content } & \multicolumn{3}{c|}{ cis-1 } & \multicolumn{2}{c|}{ trans-1 } \\
\cline { 2 - 6 } & $30 \%$ & $45 \%$ & $62 \%$ & $30 \%$ & $45 \%$ \\
\hline mesophase & $\mathrm{H}_{1}$ & $\mathrm{H}_{1}$ & $\mathrm{Ia} 3 \mathrm{~d}$ & $\mathrm{H}_{1}$ & $\mathrm{H}_{1}$ \\
$R_{p}(\mathrm{~nm})$ & 2.85 & 2.79 & 2.95 & 3.11 & 2.78 \\
$R_{h}(\mathrm{~nm})$ & 0.78 & 0.93 & 0.94 & 0.90 & 1.0 \\
$A_{m}\left(\mathrm{~nm}^{2}\right)$ & 0.84 & 0.70 & 0.69 & 0.72 & 0.65 \\
$q_{\text {max }}\left(\mathrm{nm}^{-1}\right)$ & 1.45 & 1.67 & 1.77 & 1.29 & 1.42 \\
$R_{p}=$ polar radius; $R_{h}=$ hydrophobic radius; $A_{m}=$ area at the polar/non-polar interface
\end{tabular}

At $80 \%$, the peaks corresponding to the cubic phase have smeared to form a wide band and smaller structures appear. Further in the dry sample, the band has almost completely disappeared and the peaks are neater (Figure S7 in SI). The precise structure of this solid has not been elucidated; however, several of the peaks could be indexed by a square structure with peaks ordered in the sequence $1,2^{1 / 2}, 4^{1 / 2}, 5^{1 / 2}, 8^{1 / 2} \ldots$ with some additional peaks. This arrangement could correspond to quasi square structure of cylindrical 
units. Moreover, information about the direct or inverse nature of these structures could not be determined. Because of the limitations of the used SAXS system, further research to elucidate this structure should use synchrotron radiation and better crystals.

The results for diastereoisomer trans-1 do not differ markedly from those of cis-1 at the lower concentrations (Figure S8 in SI). In fact, the scattering curves also correspond to cylinders in a loose hexagonal packing for the $30 \%$ sample and increased order is observed for the $45 \%$ sample. The peak positions, however, differ significantly. The positions for the trans-1 compound correspond to $15 \%$ thicker cylinders. In turn, this corresponds to a decrease in area per molecule and an increase in the hydrophobic length of the cylinders (Table 3). A second aspect corresponds to the degree of order in the hexagonal phase, which is higher for the trans-1 diastereoisomers, if the higher order is attributed to higher net charge. The latter would agree with the smaller $\mathrm{pK}_{\mathrm{a}}$ as determined at concentrations well above the $c m c$ (see Figure S1 in the SI). However, an increase in charge should also be associated with an increase of the area per molecule, which is not the case. On the other hand, the increase in ionic strength helps in decreasing the ion-ion repulsions. Therefore, the ionic aspects may play only a minor role in this structuring, and hydrogen bonding and steric effects could be the dominant forces. The sample with $60 \%$ showed the evolution of the structure from that of the hexagonal phase to a structure that can be considered as a swollen form of the dry structure. Concerning the slow transformation from hexagonal to the swollen dry structure, one is tempted to think of a simple rearrangement of the cylinders, however, the inversion of the cylinder structure is also possible. Neither it is clear the relationship between the dry and swollen sample. The decrease in linear distance implied by the change in peak position is close to that expected for a tridimensional isometric shrinkage, which could agree with some cubic structure. However, the observed spacing does not correspond to any of the known structures for lyotropic liquid crystals. Moreover, taking the tridimensional shrinkage as a guide, the second peak of the $60 \%$ sample should move to the right of the wide bump present in the dry sample (see the straight lines in Figure S8 in SI) while the third peak should appear at a $q \approx 5 \mathrm{~nm}^{-1}$. Otherwise, it is difficult to accommodate the observed position of the peaks to the described lyotropic crystalline structures. 
Chirality and chiral recognition in micellar aggregates of surfactants cis- and trans-1. In order to investigate the expression of chirality in surfactant cis- and trans-1 aggregates, Circular Dichroism (CD) spectra were recorded for their water solutions at concentrations both below (monomers) and above their cmc (see Figure S9 in the SI). The observed CD bands in the 190-240 nm spectral range correspond to the contribution of the Cotton effects of the amide bond and the carboxylate chromophore. ${ }^{43}$ The CD spectrum of cis-1 in non-aggregative conditions shows an intense negative monosignate band with a minimum at $206 \mathrm{~nm}$. On the other hand, the spectrum of trans-1 diastereoisomer shows an apparent positive couplet (bisignate band) centered at $200 \mathrm{~nm}$. Upon aggregation, the CD spectra did not change significantly, and only a reproducible increase of band intensity was observed for both surfactants with a slight blue shift in the case of cis-1. The latter is likely due to changes in micropolarity around the chromophores at the surface of the aggregates induced by solvation and electrostatic interactions.

It is well known that CD bands are extremely sensitive to changes in the "chiral environment" close to the chromophore to which they are allied and to interchromophoric interactions (coupling between chromophores disposed in chiral arrangements) in supramolecular aggregates. ${ }^{44}$ For example, strong changes in the sign and intensity of $\mathrm{CD}$ bands have been observed upon micellization of some $\mathrm{N}$ acylamino acid surfactants, ${ }^{40,45}$ and have been interpreted as due to the formation of an ordered chiral arrangement of amide bond planes on the micellar surface stabilized by a network of intermolecular hydrogen-bonds between adjacent molecules. Nevertheless, in many cases, changes are limited to a simple increase of intensity. ${ }^{5}$ In the present case, the absence of drastic changes in the CD spectra upon aggregation suggests the formation of loose micellar aggregates in which molecules (and thus chromophores) feature a high degree of rotational disorder. ${ }^{46} \mathrm{We}$ should remark that this finding does not preclude the chiral nature of the surfactant as being responsible for the formation of fibrils in these systems because the amount of surfactant present in the fibers corresponds only to a moderate proportion. In any case, the lack of any detectable ordered chiral arrangement of amide chromophores does not exclude the investigated surfactant aggregates, which are chiral entities, from featuring chiral recognition abilities. In fact, it has been previously shown that the expression of a chiral function, like chiral 
recognition, in surfactant aggregates depends upon many parameters and is triggered by a complex sequence of recognition processes. ${ }^{8,9}$ In some cases, it was found that micelles can discriminate the enantiomers of a chiral solute in sites in the hydrophobic domain, i.e. far from the stereogenic centres of the chiral heads. ${ }^{6}$

In order to assess the ability of the micellar aggregates of the two diastereomeric surfactants $\mathbf{1}$ to recognize chiral solutes, chiral recognition experiments were performed using bilirubin IX $\alpha(\mathrm{BR})$ as probe of chirality, which is a racemic mixture of rapidly interconverting enantiomers (see Scheme S1 in the SI). The deracemization of bilirubin (i.e. the shift of the 1:1 enantiomeric equilibrium) has been observed upon its interaction with chiral selectors such as proteins, ${ }^{47}$ chiral amines,${ }^{48}$ cyclodextrins,${ }^{49}$ and cholic salt micelles. ${ }^{50}$ Further, it was reported that micellar aggregates of anionic and cationic chiral surfactants, such as $\mathrm{N}$-acyl prolinates ${ }^{51}$ and ephedrine derivatives, ${ }^{9}$ can also induce deracemization of bilirubin. Interestingly, in these cases the extent and the direction of the enantioselection was shown to depend strongly on the length of the hydrophobic chain of the surfactant and on the concentration conditions, which affect the aggregate organization and hence the expression of chirality. ${ }^{9}$

Figure 6 (top) shows the $\mathrm{CD}$ spectra of $10 \mu \mathrm{M}$ bilirubin in the presence of micellar aggregates of surfactants cis- and trans-1 (40 mM solutions at spontaneous $\mathrm{pH})$. The CD spectrum of bilirubin in aggregates of cis $\mathbf{- 1}$ shows a bisignate band (a positive couplet) centered at the absorption maximum of the pigment. On the other hand, solutions of bilirubin in aggregates of trans-1 are almost CD silent. The occurrence of such an exciton couplet for bilirubin (in the absence of other chromophores absorbing in the same spectral region) can be safely ascribed to the deracemization of its racemic mixture. In particular, a positive couplet is associated to the preference for the $P$-enantiomer. ${ }^{52}$

The corresponding UV-Vis spectra (Figure 6 bottom) suggest that bilirubin is present as a monomer in the surfactant aggregates. Moreover, they show the two transitions of exciton splitting of comparable intensity implying a similar ridge tile conformation for pigment inside the aggregates formed by both surfactants. This fact rules out the possibility that bilirubin is adopting a flattened, CD silent conformation in aggregates of trans $-1 .{ }^{52,9}$ As a result, it can be safely claimed that the different CD spectra observed for 
bilirubin in aggregates of surfactants $\mathbf{1}$ actually results from their markedly different enantioselection ability. $^{53}$

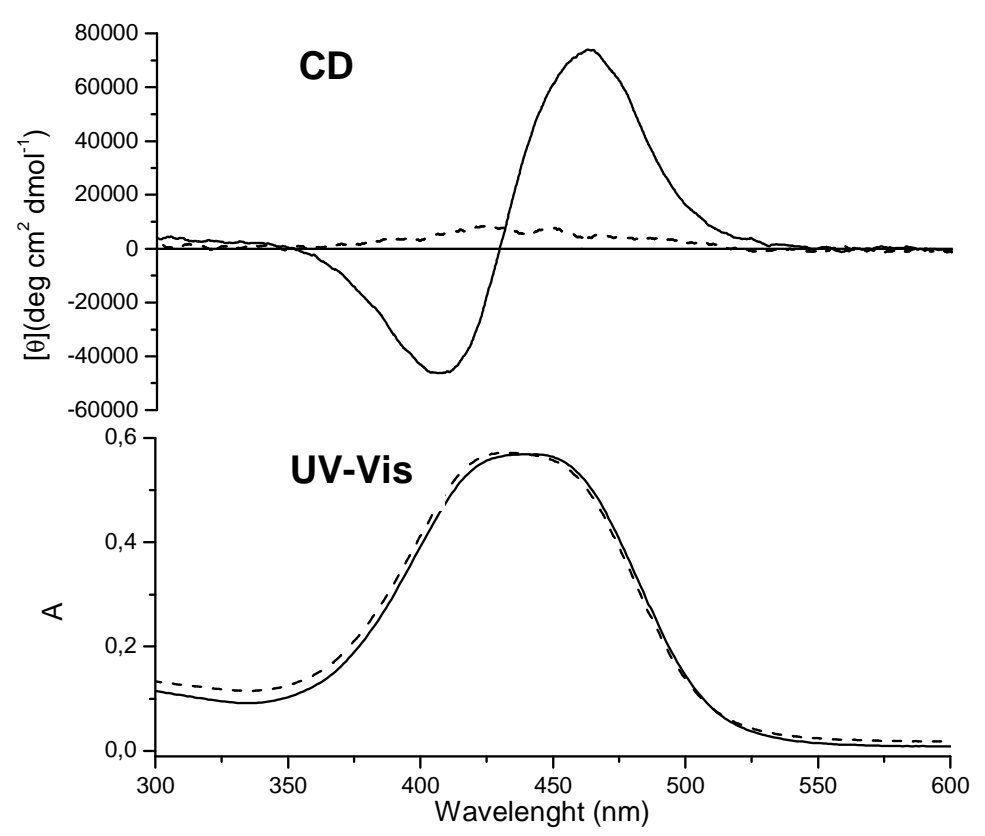

Figure 6. CD (top) and UV-Vis (bottom) spectra of $10 \mu \mathrm{M}$ BR in the presence of 40mM cis-1 (solid line) and $40 \mathrm{mM}$ trans-1 (dashed line); optical path $1 \mathrm{~cm}$.

To rule out the possibility that the observed enantioselection arises from the interaction of the chiral probe with surfactant monomers, the CD spectra of samples of bilirubin in the presence of surfactants cis- and trans-1, respectively, at high concentration $(40 \mathrm{mM})$ in methanol and below the $c m c(1.6 \mathrm{mM})$ in water, i.e. in non-aggregative conditions, were registered. While samples in methanol are almost CD silent for both surfactants, samples in water below the $c m c$ display complex CD spectra (convolution of several Cotton effects), different from that observed above the $c m c$ (Figure 7 top). In particular, the CD spectrum of bilirubin in the presence of trans-1 shows an asymmetric negative couplet with a remarkably red shifted lowest energy component. On the other hand, in the presence of cis-1, the spectrum shows a more intricate pattern, probably arising from the superimposition of two exciton couplets with opposite sign and slightly different positions, and an additional positive shoulder at $489 \mathrm{~nm}^{54}$ 


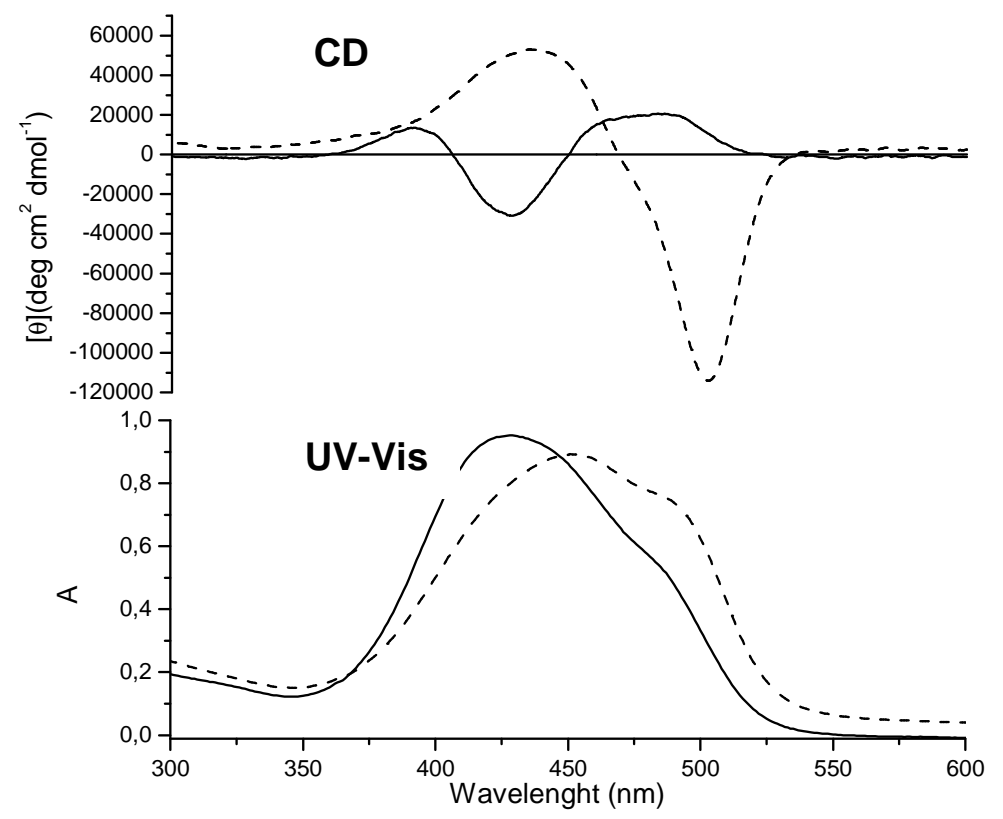

Figure 7. CD (top) and UV-Vis (bottom) spectra of $20 \mu \mathrm{M}$ BR in the presence of $1.6 \mathrm{mM}$ cis-1 (solid line) and $2 \mathrm{mM}$ trans-1 (dashed line).

These spectral features suggest that the optical activity, at surfactant concentration below the $c m c$, does not arise from the enantioselective interaction of bilirubin with the chiral surfactant as a monomer. More likely, it results from the formation of surfactant/bilirubin heteroaggregates featuring enantiodiscrimination, evidently induced by the homochiral surfactants. Actually, the strong red shifted CD band in the presence of trans-1, as well as the component at $489 \mathrm{~nm}$ in the presence of cis-1, jointly with the increase in absorption at about $500 \mathrm{~nm}$ in the corresponding UV-Vis spectra (Figure 7 bottom), indicate the occurrence of inter chromophore interactions inside the aggregates (i.e. intermolecular electric dipole coupling between bilirubin molecules). A similar kind of heteroaggregates responsible for enantioselection has been previously reported for the interaction of bilirubin with cholic salts (anionic amphiphiles) at concentrations below their $c m c .{ }^{50}$ In the present case, it should be stressed that the mode of interaction of the diastereomeric surfactants $\mathbf{1}$ with bilirubin at submicellar concentrations is rather different. In fact, while trans-1 likely induces the formation of heteroaggregates in which deracemization occurs with a preference for $M$-enantiomer (enantioselection), deracemization is not clearly observed in the case of the interaction of bilirubin with cis-1. A possibility is that $\boldsymbol{c i s - 1}$ forms heteroaggregates with racemic (or almost racemic) bilirubin in which the two enantiomers ( $M$ and $P$ ) feature non perfectly 
mirror image CD spectra due to their diastereomeric interactions with the chiral surfactant, and maybe different conformations. Thus, a non-vanishing CD spectrum is obtained even in the presence of a racemate as a result of enantiodiscrimination. ${ }^{55}$ Note that similar results below the $\mathrm{cmc}$ were also obtained working at $\mathrm{pH} 8.7$ (diluted $\mathrm{NaOH}$ solutions) chosen to simulate the spontaneous $\mathrm{pH}$ of micellar solutions. This excludes the possibility that the different behavior below and above the $c m c$ depends on a different state of charge of the pigment. Moreover, it strengthens the hypothesis that the spectra below the $\mathrm{cmc}$ are due to surfactant/bilirubin heteroassociation, rather than to bilirubin diacid self-aggregation, which has been observed at neutral and acid $\mathrm{pH}$. As a matter of fact, the formation of surfactant/bilirubin heteroaggregates might also explain the surprising resistance to photo bleaching observed for samples at surfactant concentration below the $c m c$. Indeed, their UV-Vis spectra remained unchanged after a week of exposure to direct light, while it is known that bilirubin monomers undergo rapid photodegradation even under dim light.

To sum up, the stereochemistry of the anionic surfactants cis- and trans-1 has a dramatic influence on the extent of enantioselection, as well as on the fashion in which chirality is expressed at the supramolecular level, by controlling the molecular organization both in micellar aggregates as well as in the surfactant/bilirubin heteroaggregates.

\section{CONCLUSIONS}

Through the systematic comparison of cis and trans diastereoisomers of a new family of amphiphiles based on the cyclobutane $\beta$-amino acid structure, we have shown the relevance of the relative stereochemistry and of the stereochemical constraints imposed by the cyclobutane ring on the aggregation properties of these compounds at different aggregation states. In diluted micellar solutions of the anionic surfactants cis- and trans-1, the general outcome from the different techniques can be rationalized on the basis of the influence of stereochemistry on the anionic charge stabilization and headgroup solvation. Namely, the results suggest a better stabilization of the anionic charge in the case of surfactant cis-1, likely due to intra-molecular hydrogen-bonding and/or charge-dipole interactions. This causes differences in $\mathrm{pK}_{\mathrm{a}}, c m c$, and conductivity, as well as in the structural parameters of the spherical micelles at small 
concentrations (number of aggregation, dimensions, hydrophobic radius). However, the effect of the stereochemical constraints (the cyclobutane ring restrains the amide and carboxylate groups at a fixed relative orientation) should be invoked to explain the different morphology and kinetics of formation of the insoluble long fibers that remarkably evolve with time from the spherical micelles of the two surfactants cis- and trans-1. Indeed, such constraints influence the self-organization of molecules within the aggregates and the possibility to form networks of directional intermolecular hydrogen-bonds at the aggregate surface, which most probably drives the slow fiber formation. In the lyotropic liquid crystal domain, the differences at intermediate concentrations are still significant, but not remarkable, while at higher concentration, up to the dry state, they become larger again with different mesophases observed for the two diastereomeric surfactants. These differences can hardly be attributed to head charge effects but have to be ascribed to different hydrogen-bond patterns and molecular packing induced by the different relative stereochemistry.

Noteworthy, the spherical micellar aggregates of the two surfactants cis-and trans-1 feature dramatically different enantioselection ability for bilirubin enantiomers. In fact, the different configuration of these surfactants by controlling the molecular organization and structure of micellar aggregates (albeit they are mainly loose dynamic assemblies) can affect their chiral recognition ability. In addition, it also influences the fashion in which chirality is expressed at the supramolecular level of surfactant/bilirubin heteroaggregates formed at submicellar concentrations of surfactants cis- and trans-1. This confirms that the expression of chirality in dynamic assemblies of amphiphiles is triggered by a complex sequence of recognition processes that depend on the organization of the whole assembly. ${ }^{56}$

All these differential features should allow the rational design of systems with predictable behavior and tunable properties, which must be useful in the development of new soft materials. With this main goal in mind, active investigations are being carried out in our laboratories.

\section{ASSOCIATED CONTENT}

\section{Supporting information}


Synthesis and characterization of surfactants $\mathbf{1}$ and their precursors, ${ }^{1} \mathrm{H}$ and ${ }^{13} \mathrm{C}$ NMR spectra of new compounds, details on $\mathrm{pH}$ measurements, SAXS measurement in diluted (micellar) solution and lyotropic liquid crystals of surfactants 1, TEM and SEM images of fibrous assemblies formed by surfactants, optical micrograph of fibrous assemblies formed by surfactants 1, SAXS measurements of lyotropic liquid crystals of surfactants $\mathbf{1}, \mathrm{CD}$ Spectra of surfactants $\mathbf{1}$ and their aggregates, bilirubin molecular structure. This material is available free of charge via the Internet at http://pubs.acs.org

\section{AUTHOR INFORMATION}

\section{Corresponding authors}

Rosa.ortuno@uab.es, ramon.pons@iqac.csic.es

\section{ACKNOWLEDGMENTS}

We thank Jaume Caelles, in the SAXS-WAXS service at IQAC, for X-Ray measurements, and Imma Carrera for technical assistance in the surface tension measurements. Financial support from MINECO (grants CTQ2010-15408/BQU, CTQ2013-41514-P, and CTQ2013-43754-P) is gratefully acknowledged. Authors also thank the support from Generalitat de Catalunya (2014SGR358 and 2014SGR836).

\section{REFERENCES}

(1) Sorrenti, A.; Illa, O.; Ortuño, R. M. Amphiphiles in Aqueous Solution: Well beyond a Soap Bubble. Chem. Soc. Rev. 2013, 42, 8200-8219.

(2) Fuhrhop, J.-H.; Koning, J.; Royal Society of Chemistry (UK). Membranes and Molecular Assemblies : The Synkinetic Approach; Monographs in supramolecular chemistry; Royal Society of Chemistry: Cambridge, 1994.

(3) Menger, F. M. Supramolecular Chemistry and Self-Assembly. Proc. Natl. Acad. Sci. 2002, 99, 48184822.

(4) Estroff, L. A.; Hamilton, A. D. Water Gelation by Small Organic Molecules. Chem. Rev. 2004, 104, $1201-1218$.

(5) Brizard, A.; Oda, R.; Huc, I. Chirality Effects in Self-Assembled Fibrillar Networks. Top. Curr. Chem.. 2005, 256, 167-218. 
(6) Bombelli, C.; Borocci, S.; Lupi, F.; Mancini, G.; Mannina, L.; Segre, A. L.; Viel, S. Chiral Recognition of Dipeptides in a Biomembrane Model. J. Am. Chem. Soc. 2004, 126, 13354-13362.

(7) Nandi, N.; Vollhardt, D. Effect of Molecular Chirality on the Morphology of Biomimetic Langmuir Monolayers. Chem. Rev. 2003, 103, 4033-4075.

(8) Ceccacci, F.; Mancini, G.; Sferrazza, A.; Villani, C. pH Variation as the Switch for Chiral Recognition in a Biomembrane Model. J. Am. Chem. Soc. 2005, 127, 13762-13763.

(9) Bombelli, C.; Bernardini, C.; Elemento, G.; Mancini, G.; Sorrenti, A.; Villani, C. Concentration as the Switch for Chiral Recognition in Biomembrane Models. J. Am. Chem. Soc. 2008, 130, 2732-2733.

(10) Petkovich, N. D.; Stein, A. Controlling Macro- and Mesostructures with Hierarchical Porosity through Combined Hard and Soft Templating. Chem. Soc. Rev. 2013, 42, 3721-3739.

(11) Che, S.; Liu, Z.; Ohsuna, T.; Sakamoto, K.; Terasaki, O.; Tatsumi, T. Synthesis and Characterization of Chiral Mesoporous Silica. Nature 2004, 429, 281-284.

(12) Liu, Y.; Goebl, J.; Yin, Y. Templated Synthesis of Nanostructured Materials. Chem. Soc. Rev. 2013, 42 , $2610-2653$.

(13) Van Bommel, K. J. C.; Friggeri, A.; Shinkai, S. Organic Templates for the Generation of Inorganic Materials. Angew. Chem. Int. Ed. 2003, 42, 980-999.

(14) Hartgerink, J. D.; Beniash, E.; Stupp, S. I. Self-Assembly and Mineralization of Peptide-Amphiphile Nanofibers. Science 2001, 294, 1684-1688.

(15) Luk, Y.-Y.; Abbott, N. L. Applications of Functional Surfactants. Curr. Opin. Colloid Interface Sci. 2002, 7, 267-275.

(16) Soussan, E.; Cassel, S.; Blanzat, M.; Rico-Lattes, I. Drug Delivery by Soft Matter: Matrix and Vesicular Carriers. Angew. Chem. Int. Ed. 2009, 48, 274-288.

(17) Berti, D. Self-Assembly of Biologically Inspired Amphiphiles. Curr. Opin. Colloid Interface Sci. 2006, $11,74-78$.

(18) Pinazo, A.; Pons, R.; Pérez, L.; Infante, M. R. Amino Acids as Raw Material for Biocompatible Surfactants. Ind. Eng. Chem. Res. 2011, 50, 4805-4817.

(19) Infante, M. R.; Pérez, L.; Morán, M. C.; Pons, R.; Mitjans, M.; Vinardell, M. P.; Garcia, M. T.; Pinazo, 
A. Biocompatible Surfactants from Renewable Hydrophiles. Eur. J. Lipid Sci. Technol. 2010, 112, 110-121.

(20) Gorrea, E.; Pohl, G.; Nolis, P.; Celis, S.; Burusco, K. K.; Branchadell, V.; Perczel, A.; Ortuño, R. M. Secondary Structure of Short $\beta$-Peptides as the Chiral Expression of Monomeric Building Units: A Rational and Predictive Model. J. Org. Chem. 2012, 77, 9795-9806.

(21) Celis, S.; Nolis, P.; Illa, O.; Branchadell, V.; Ortuño, R. M. Low-Molecular-Weight Gelators Consisting of Hybrid Cyclobutane-Based Peptides. Org. Biomol. Chem. 2013, 11, 2839-2846.

(22) Torres, E.; Puigmarti-Luis, J.; Perez del Pino, A.; Ortuño, R. M.; Amabilino, D. B. Use of Unnatural $\beta$ Peptides as a Self-Assembling Component in Functional Organic Fibres. Org. Biomol. Chem. 2010, 8, 16611665.

(23) Torres, E.; Gorrea, E.; Burusco, K. K.; Da Silva, E.; Nolis, P.; Rua, F.; Boussert, S.; Diez-Perez, I.; Dannenberg, S.; Izquierdo, S.; Giralt, E.; Jaime, C.; Branchadell, V.; Ortuño, R. M. Folding and SelfAssembling with $\beta$-Oligomers Based on (1R,2S)-2-Aminocyclobutane-1-Carboxylic Acid. Org. Biomol. Chem. 2010, 8, 564-575.

(24) Fernandes, C.; Faure, S.; Pereira, E.; Théry, V.; Declerck, V.; Guillot, R.; Aitken, D. J. 12-Helix Folding of Cyclobutane $\beta$-Amino Acid Oligomers. Org. Lett. 2010, 12, 3606-3609.

(25) Gorrea, E.; Nolis, P.; Torres, E.; Da Silva, E.; Amabilino, D. B.; Branchadell, V.; Ortuño, R. M. SelfAssembly of Chiral Trans-Cyclobutane-Containing $\beta$-Dipeptides into Ordered Aggregates. Chem. - Eur. J. 2011, 17, 4588-4597.

(26) S.H. Anastasiadis, S.H.; Chen, J.-K.; Koberstein, J.T.; Siegel, A.F.; Sohn, J.E.; Emerson, J.A. The determination of interfacial tension by video image processing of pendant fluid drops. J. Colloid Interface Sci. 1987, 119, 55-66.

(27) D. Orthaber; A. Bergmann; O. Glatter. SAXS Experiments on Absolute Scale with Kratky Systems Using Water as a Secondary Standard. J. Appl. Crystallogr. 2000, 33, 218.

(28) Perez, L.; Pinazo, A.; Infante, M. R.; Pons, R. Investigation of the Micellization Process of Single and Gemini Surfactants from Arginine by SAXS, NMR Self-Diffusion, and Light Scattering. J. Phys. Chem. B 2007, 111, 11379-11387.

(29) Pedersen, J. S. Analysis of Small-Angle Scattering Data from Colloids and Polymer Solutions: 
Modeling and Least-Squares Fitting. Adv. Colloid Interface Sci. 1997, 70, 171-210.

(30) Pons, R.; Valiente, M.; Montalvo, G. Structure of Aggregates in Diluted Aqueous Octyl Glucoside/Tetraethylene Glycol Monododecyl Ether Mixtures with Different Alkanols. Langmuir 2010, 26, $2256-2262$.

(31) Pedersen, J. Form Factors of Block Copolymer Micelles with Spherical, Ellipsoidal and Cylindrical Cores. J. Appl. Crystallogr. 2000, 33, 637-640.

(32) Förster, S.; Timmann, A.; Konrad, M.; Schellbach, C.; Meyer, A.; Funari, S. S.; Mulvaney, P.; Knott, R. Scattering Curves of Ordered Mesoscopic Materials. J. Phys. Chem. B 2005, 109, 1347-1360.

(33) Bellamy, L. J. The Infra-Red Spectra of Complex Molecules, 3d ed.; Chapman and Hall ; Wiley: London New York, 1975.

(34) Miles, G. D. Minima in Surface-Tension and Interfacial-Tension Curves. J. Phys. Chem. 1945, 49, 7176.

(35) Xu, H.; Li, P. X.; Ma, K.; Thomas, R. K.; Penfold, J.; Lu, J. R. Limitations in the Application of the Gibbs Equation to Anionic Surfactants at the Air/water Surface: Sodium Dodecylsulfate and Sodium Dodecylmonooxyethylenesulfate above and below the CMC. Langmuir 2013, 29 , 9335-9351.

(36) Hiemenz, P. C.; Rajagopalan, R. Principles of Colloid and Surface Chemistry, 3rd ed.; Marcel Dekker: New York, 1997.

(37) Rosen, M. J. Surfactants and Interfacial Phenomena, 3rd ed.; Wiley-Interscience: Hoboken, N.J., 2004.

(38) Menger, F. M.; Caran, K. L. Anatomy of a Gel. Amino Acid Derivatives That Rigidify Water at Submillimolar Concentrations. J. Am. Chem. Soc. 2000, 122, 11679-11691.

(39) Imae, T.; Takahashi, Y.; Muramatsu, H. Formation of Fibrous Molecular Assemblies by Amino-Acid Surfactants in Water. J. Am. Chem. Soc. 1992, 114, 3414-3419.

(40) Mohanty, A.; Dey, J. Effect of the Headgroup Structure on the Aggregation Behavior and Stability of Self-Assemblies of Sodium N-[4-(n-Dodecyloxy)benzoyl]-L-Aminoacidates in Water. Langmuir 2007, 23, 1033-1040.

(41) Fuhrhop, J. H.; Helfrich, W. Fluid and Solid Fibers Made of Lipid Molecular Bilayers. Chem. Rev. 1993, 93, 1565-1582. 
(42) Garstecki, P.; Hołyst, R. Scattering Patterns of Self-Assembled Cubic Phases. 1. The Model. Langmuir 2002, 18, 2519-2528.

(43) Berova, N.; Nakanishi, K.; Woody, R. W. Circular Dichroism: Principles and Applications, 2nd Edition; Wiley: New York, 2001.

(44) Berova, N.; Di Bari, L.; Pescitelli, G. Application of Electronic Circular Dichroism in Configurational and Conformational Analysis of Organic Compounds. Chem. Soc. Rev. 2007, 36, 914-931.

(45) Shinitzky, M.; Haimovitz, R. Chiral Surfaces in Micelles of Enantiomeric N-Palmitoyl- and NStearoylserine. J. Am. Chem. Soc. 1993, 115, 12545-12549.

(46) It has to be remarked that the contribution of the surfactant monomers to the CD spectrum is relevant at the investigated concentrations. On the other hand, the investigation at higher concentrations was precluded by the strong absorbance of the surfactants.

(47) Blauer, G. Complexes of Bilirubin with Proteins. Biochim. Biophys. Acta (BBA) 1986, 884, 602-604.

(48) Lightner, D. A.; Gawronski, J. K.; Wijekoon, W. M. D. Complementarity and Chiral Recognition: Enantioselective Complexation of Bilirubin. J. Am. Chem. Soc. 1987, 109, 6354-6362.

(49) Lightner, D. A.; Gawronski, J. K.; Gawronska, K. Conformational Enantiomerism in Bilirubin. Selection by Cyclodextrins. J. Am. Chem. Soc. 1985, 107, 2456-2461.

(50) D’Alagni, M.; D’Archivio, A. A.; Giglio, E.; Scaramuzza, L. Structure of Sodium and Rubidium Taurodeoxycholate Micellar Aggregates and Their Interaction Complexes with Bilirubin-IX.alpha. J. Phys. Chem. 1994, 98, 343-353.

(51) Ceccacci, F.; Giansanti, L.; Mortera, S. L.; Mancini, G.; Sorrenti, A.; Villani, C. Enantiodiscrimination of Bilirubin-IX $\alpha$ Enantiomers in Biomembrane Models: Has Chirality a Role in Bilirubin Toxicity? Bioorg. Chem. 2008, 36, 252-254.

(52) Person, R. V.; Peterson, B. R.; Lightner, D. A. Bilirubin Conformational Analysis and Circular Dichroism. J. Am. Chem. Soc. 1994, 116, 42-59.

(53) Interestingly, in the case of surfactant cis-1 the intensity of the CD couplet decreases upon increasing bilirubin concentration (almost halved at $100 \mu \mathrm{M}$ BR) without substantial changes in the shape of the UV-Vis spectra (not shown) which would imply a different extent of deracemization depending on the concentration 
conditions.

(54) Note that the position of the negative Cotton effect at $428 \mathrm{~nm}$ in the CD spectrum of cis-1 coincides with the maximum in the UV-Vis spectrum which excludes the presence of a simple couplet according to the theory of exciton coupling.

(55) Krois, D.; Lehner, H. Induced Circular Dichroism and Chiral Discrimination of Racemates Revisited: Bilirubins as Illustrative Examples. J. Chem. Soc. Perkin Trans. 2 1995, 489-494.

(56) Marinelli, F.; Sorrenti, A.; Corvaglia, V.; Leone, V.; Mancini, G. Molecular Description of the Propagation of Chirality from Molecules to Complex Systems: Different Mechanisms Controlled by Hydrophobic Interactions. Chem.- Eur. J. 2012, 18, 14680-14688. 


\title{
Supporting Information
}

\section{Chiral Cyclobutane ß-Amino Acid-Based Amphiphiles: Influence of cis/trans Stereochemistry on} Solution Self-aggregation and Recognition

\author{
Alessandro Sorrenti ${ }^{1}$, Ona Illa ${ }^{1}$, Ramon Pons ${ }^{2, *}$, Rosa M. Ortuño ${ }^{1, *}$. \\ ${ }^{1}$ Departament de Quimica, Universitat Autònoma de Barcelona, 08193, Cerdanyola del Vallès, Barcelona, Spain \\ ${ }^{2}$ Departament de Tecnologia Química i de Tensioactius, Institut de Química Avançada de Catalunya, IQAC-CSIC, \\ c/Jordi Girona 18-26, 08034 Barcelona, Spain
}

\section{Table of contents}

Synthesis and characterization of $N$-lauroyl surfactants 1 and their precursors 2

${ }^{1} \mathrm{H}$ and ${ }^{13} \mathrm{C}$ NMR spectra of surfactants cis-1 and trans-1 and their precursors $\quad 7$

$\mathrm{pH}$ measurements

SAXS measurement in diluted (micellar) solution of surfactants 1

TEM and SEM images of fibrous assemblies formed by surfactants

Optical micrograph of fibrous assemblies formed by surfactants 1

SAXS measurements of lyotropic liquid crystals of surfactants 1

CD Spectra of surfactants $\mathbf{1}$ and their aggregates 


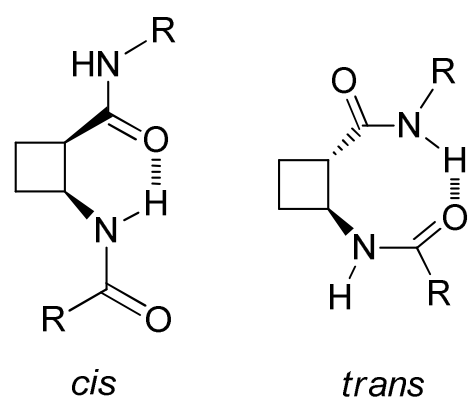

Chart S1. Different hydrogen-bonding in cis- and trans-cyclobutane monomers

\section{$\underline{\text { Synthesis and characterization of } N \text {-lauroyl surfactants } 1 \text { and their precursors }}$}

Materials and Methods. Tetrahydrofuran (THF) was freshly distilled under a nitrogen atmosphere from sodium/benzophenone. Acetone and dichloromethane $\left(\mathrm{CH}_{2} \mathrm{Cl}_{2}\right)$ were freshly distilled from calcium chloride. All other chemicals were of commercial grade and used without further purification unless otherwise stated. ${ }^{1} \mathrm{H}$ NMR and ${ }^{13} \mathrm{C}$ NMR spectra were carried out in deuterated solvents on Bruker Avance 250, 360 and $600 \mathrm{MHz}$ Ultrashield spectrometers. High-resolution mass spectra were recorded using a direct inlet system (ESI). IR spectra were obtained from pure solid samples with an ATR (Attenuated Total Reflectance) accessory. Melting points were recorded on a Reicher Klofler block and values are uncorrected. Purification of the reaction mixtures was performed by column flash chromatography with neutral silica gel (230-400 mesh).

(1R,2S)-Methyl-2-(dodecanamido)cyclobutanecarboxylate (cis-3). To an ice-cooled solution of tert-butyl $(1 S, 2 R)$-2-(methoxycarbonyl)cyclobutylcarbamate, cis-2, $(0.30 \mathrm{~g}, 1.3 \mathrm{mmol})$ in dry $\mathrm{CH}_{2} \mathrm{Cl}_{2}(7 \mathrm{ml})$, TFA $(1.29 \mathrm{ml}, 16.9 \mathrm{mmol}, 13 \mathrm{eq})$ and $\mathrm{Et}_{3} \mathrm{SiH}(0.63 \mathrm{ml}, 3.9 \mathrm{mmol}, 3 \mathrm{eq})$ were added under nitrogen atmosphere. The mixture was stirred at $0{ }^{\circ} \mathrm{C}$ for $3 \mathrm{~h}$, afterwards, it was concentrated to obtain the desired salt (quantitative yield) as a pale yellow oil which was used in the next step without purification.

To an ice cooled solution of the cyclobutylamonium trifluoroacetate salt $(0.31 \mathrm{~g}, 1.3 \mathrm{mmol})$ and lauroyl chloride (0.33 ml, $1.43 \mathrm{mmol}, 1.1 \mathrm{eq})$ in $\mathrm{CH}_{2} \mathrm{Cl}_{2}(15 \mathrm{ml}), \mathrm{Et}_{3} \mathrm{~N}(0.73 \mathrm{ml}, 5.3 \mathrm{mmol}, 4 \mathrm{eq})$ was added dropwise. The mixture was stirred at $0{ }^{\circ} \mathrm{C}$, for $5 \mathrm{~h}$ while monitoring the progress of the reaction by TLC 
(EtOAc/hexanes 2:3 as eluent). After that, more $\mathrm{CH}_{2} \mathrm{Cl}_{2}$ was added and the organic phase washed with $\mathrm{H}_{2} \mathrm{O}$ and brine, dried over $\mathrm{MgSO}_{4}$ and concentrated to give a white-yellow solid that was purified by flash chromatography using EtOAc/Hexanes 3:7 as eluent. Crystallization from $\mathrm{CH}_{2} \mathrm{Cl}_{2}$ /pentane (vapour diffusion) yielded $0.31 \mathrm{~g}(1.0 \mathrm{mmol}, 77 \%$ yield $)$ of a white crystalline solid, m.p. $61{ }^{\circ} \mathrm{C}$ (from pentane). $[\alpha]_{\mathrm{D}}^{20}-79.6\left(c\right.$ 0.7, $\left.\mathrm{CHCl}_{3}\right)$. IR $3254.4(\mathrm{NH}), 2919.5,2851.0(\mathrm{CH} \mathrm{st}), 1723.8(\mathrm{C}=\mathrm{O}), 1642.8$ (amide I), 1538.9 (amide II) $\mathrm{cm}^{-1} .{ }^{1} \mathrm{H}$ NMR $\left(360 \mathrm{MHz}, \mathrm{CDCl}_{3}\right) \delta 0.86(\mathrm{t}, 3 \mathrm{H}, J=6.8 \mathrm{~Hz}), 1.24$ (br m, 16H), 1.57 (br m, 2H), 1.90-2.42 (complex absorption, 6H), $3.38(\mathrm{~m}, 2 \mathrm{H}), 3.69(\mathrm{~s}, 3 \mathrm{H}), 4.78$ (quint, $1 \mathrm{H}, J=8.7 \mathrm{~Hz}$ ), 6.47 (br d, 1H) ppm. ${ }^{13} \mathrm{C}$ NMR $\left(90 \mathrm{MHz}, \mathrm{CDCl}_{3}\right) \delta 14.2\left(\mathrm{CH}_{3}\right), 19.3,22.8,25.8,29.5-29.7,32.0,36.9$ $\left(\mathrm{CH}_{2}\right.$ chain and cyclobutane), $43.9(\mathrm{CH}), 44.7(\mathrm{CH}), 51.8\left(\mathrm{CH}_{3}\right), 172.5(\mathrm{C}), 175.3(\mathrm{C}) \mathrm{ppm} . \mathrm{HRMS}(\mathrm{ESI}+)$ Calcd. Mass for $\mathrm{C}_{18} \mathrm{H}_{33} \mathrm{NO}_{3} \mathrm{Na}[\mathrm{M}+\mathrm{Na}]^{+}$: 334.2353; Found: 334.2361. Anal. Calcd: C 69.41; H 10.68; N 4.50. Found: C, 69.40; H 10.65; N 4.59.

(1R,2S)-2-(Dodecanamido)cyclobutanecarboxylic acid (cis-4). A solution of cis-3 (0.29 g, $0.93 \mathrm{mmol})$ in $15 \mathrm{ml}$ of a THF/ $\mathrm{H}_{2} \mathrm{O} 2: 1$ mixture was cooled at $0{ }^{\circ} \mathrm{C}$ and $10.2 \mathrm{ml}$ of $0.25 \mathrm{M} \mathrm{NaOH}(2.55 \mathrm{mmol}, 2.7 \mathrm{eq})$ was added. The mixture was stirred $5 \mathrm{~h}$ from $0{ }^{\circ} \mathrm{C}$ to room temperature, after that it was acidified to $\mathrm{pH} 2$ by $1 \mathrm{M} \mathrm{HCl}$ and extracted with $\mathrm{CH}_{2} \mathrm{Cl}_{2}$. The organic phase was dried over $\mathrm{MgSO}_{4}$ and concentrated, under reduced pressure, to give $0.27 \mathrm{~g}(0.90 \mathrm{mmol}, 97 \%$ yield $)$ as a white crystalline solid. Crystals, m.p. $67-68{ }^{\circ} \mathrm{C}$ (from pentane). $[\alpha]_{\mathrm{D}}{ }^{20}-98.4\left(c\right.$ 0.5, $\left.\mathrm{CH}_{3} \mathrm{OH}\right)$. IR 3397.9 and $3280.9(\mathrm{NH}), 2916.8,2848.7(\mathrm{CH}$ st), $1722.0(\mathrm{C}=\mathrm{O}), 1639.8$ (amide I), 1540.5 (amide II) $\mathrm{cm}^{-1} .{ }^{1} \mathrm{H}$ NMR $\left(250 \mathrm{MHz}, \mathrm{CDCl}_{3}\right) \delta 0.87(\mathrm{t}, 3 \mathrm{H}, J$ $=6.3 \mathrm{~Hz}$ ), 1.25 (br m, 16H), 1.59 (br m, 2H), 2.00-2.50 (complex absorption, 6H), 3.45 (m, 2H), 4.78 (m, 1H), 6.62 (br d, 1H) ppm. ${ }^{13} \mathrm{C}$ NMR $\left(62.5 \mathrm{MHz}, \mathrm{CDCl}_{3}\right) \delta 14.3\left(\mathrm{CH}_{3}\right), 19.4,22.8,25.8,29.4-29.8,32.0$, $36.8\left(\mathrm{CH}_{2}\right.$ chain and cyclobutane), $44.2(\mathrm{CH}), 44.8(\mathrm{CH}), 173.5(\mathrm{C}), 177.8(\mathrm{C})$ ppm. HRMS (ESI+) Calcd. Mass for $\mathrm{C}_{17} \mathrm{H}_{31} \mathrm{NO}_{3} \mathrm{Na}[\mathrm{M}+\mathrm{Na}]^{+}:$320.2196. Found: 320.2209 .

Sodium (1R,2S)-2-(dodecanamido)cyclobutanecarboxylate (cis-1). The sodium salt was prepared by adapting a previously reported procedure [Roy, S.; Dey, J. Langmuir 2005, 21, 10362]. The intermediate acid cis-2 (0.277 g, $0.93 \mathrm{mmol})$ was dissolved in $10 \mathrm{ml}$ of $\mathrm{THF} / \mathrm{H}_{2} \mathrm{O} 1: 1 \mathrm{mixture}$, then $\mathrm{NaHCO}_{3}(0.078 \mathrm{~g}$, $0.93 \mathrm{mmol}, 1$ eq.) was added as a solid, the mixture was sonicated and left under stirring for $4 \mathrm{~h}$ 
(evolution of carbon dioxide was clearly visible). After that, it was freeze-dried and the solid recrystallized twice from THF obtaining $0.238 \mathrm{~g}(0.74 \mathrm{mmol}, 80 \%$ yield over 2 crystallizations $)$ of a white crystalline solid. m.p. $145-150{ }^{\circ} \mathrm{C}$ (from THF). $[\alpha]_{\mathrm{D}}{ }^{20}-11.3\left(c 0.5, \mathrm{CH}_{3} \mathrm{OH}\right)$. IR $3340.6(\mathrm{NH})$, 2919.3, 2850.1 (CH st), 1620.1 (amide I), $1573.6\left(\mathrm{COO}^{-}\right), 1518.9$ (amide II), $1414.9\left(\mathrm{COO}^{-}\right) \mathrm{cm}^{-1} .{ }^{1} \mathrm{H}$ NMR (360 MHz, CD 3 OD) $\delta 0.90(\mathrm{t}, 3 \mathrm{H}, J=6.6 \mathrm{~Hz}), 1.29$ (br m, 16H), 1.59 (br m, 2H), 1.92-2.34 (complex absorption, 6H), $3.16(\mathrm{~m}, 1 \mathrm{H}), 4.45(\mathrm{q}, 1 \mathrm{H}, J=8.1 \mathrm{~Hz}) \mathrm{ppm} .{ }^{13} \mathrm{C} \mathrm{NMR}\left(90 \mathrm{MHz}, \mathrm{CD}_{3} \mathrm{OD}\right) \delta$ $14.4\left(\mathrm{CH}_{3}\right), 21.6,23.7,26.9,30.3-30.7,33.1,37.6\left(\mathrm{CH}_{2}\right.$ chain and cyclobutane $), 46.2(\mathrm{CH}), 47.0(\mathrm{CH})$, 174.9 (C), 181.8 (C) ppm. HRMS (ESI-) Calcd. Mass for $\mathrm{C}_{17} \mathrm{H}_{30} \mathrm{NO}_{3}{ }^{-}$[M-Na] $]^{-}$296.2231. Found: 296.2224. Anal. Calcd: C 63.92; H 9.47; N 4.39. Found: C 64.18; H 9.69; N 4.26.

When not differently specified, the trans amphiphiles (trans-1,3,4) were prepared according to the same procedures as for the cis analogues.

(1S,2S)-Methyl-2-(dodecanamido)cyclobutanecarboxylate (trans-3). Starting from $0.241 \mathrm{~g}$ (1.05 mmol) of tert-butyl $(1 S, 2 S)$-2-(methoxycarbonyl)cyclobutylcarbamate, trans-2, the trans ammonium salt was prepared as reported above, obtaining $0.255 \mathrm{~g}$ of a pale yellow oil (quantitative yield), which was used in the next step without purification.

The cyclobutylamonium trifluoroacetate salt $(0.265 \mathrm{~g}, 1.09 \mathrm{mmol})$ was acylated with lauroyl chloride $(0.28 \mathrm{ml}, 1.2 \mathrm{mmol}, 1.1 \mathrm{eq})$ according to the procedure described above for cis-3. Purification by flash chromatography using EtOAc/hexanes 3:7 as eluent followed by crystallization from $\mathrm{CH}_{2} \mathrm{Cl}_{2} /$ pentane (vapor diffusion) yielded $0.225 \mathrm{~g}\left(66 \%\right.$ yield) of a white solid. m.p. $80-81{ }^{\circ} \mathrm{C}$ (from pentane). $[\alpha]_{\mathrm{D}}{ }^{20}+$ 57.0 ( $c$ 0.7, $\mathrm{CHCl}_{3}$ ). IR $3287.9(\mathrm{NH}), 2919.2,2848.7$ (CH st), 1734.7 (C=O), 1645.5 (amide I), 1547.6 (amide II) $\mathrm{cm}^{-1} .{ }^{1} \mathrm{H}$ NMR $\left(250 \mathrm{MHz}, \mathrm{CDCl}_{3}\right) \delta 0.87(\mathrm{t}, 3 \mathrm{H}, J=6.6 \mathrm{~Hz}), 1.25$ (br m, 16H), 1.59 (br m, 2H), 1.79-2.40 (complex absorption, 6H), 3.01 (q, 1H, $J=9.0 \mathrm{~Hz}), 3.68(\mathrm{~s}, 3 \mathrm{H}), 4.48(\mathrm{~m}, 1 \mathrm{H}), 5.65$ (br s, 1H) ppm. ${ }^{13} \mathrm{C}$ NMR $\left(90 \mathrm{MHz}, \mathrm{CDCl}_{3}\right) \delta 14.2\left(\mathrm{CH}_{3}\right), 18.7,22.8,25.7,27.3,29.4-29.7,32.0,36.8\left(\mathrm{CH}_{2}\right.$ chain and cyclobutane), $46.7(\mathrm{CH}), 47.8(\mathrm{CH}), 52.0\left(\mathrm{CH}_{3}\right), 172.7(\mathrm{C}), 173.4(\mathrm{C})$ ppm. HRMS (ESI+) Calcd. Mass for $\mathrm{C}_{18} \mathrm{H}_{33} \mathrm{NO}_{3} \mathrm{Na}[\mathrm{M}+\mathrm{Na}]^{+}:$334.2353. Found: 334.2352. Anal. Calcd: $\mathrm{C} 69.41 ; \mathrm{H} 10.68 ; \mathrm{N}$ 4.50. Found: C, 69.31; H 10.76; N 4.51. 
(1S,2S)-2-(Dodecanamido)cyclobutanecarboxylic acid (trans-4). A solution of trans-3 (0.180 g, 0.58 mmol) in $9 \mathrm{ml}$ of a $\mathrm{THF} / \mathrm{H}_{2} \mathrm{O} 2: 1$ mixture was cooled at $0{ }^{\circ} \mathrm{C}$ and $6 \mathrm{ml}$ of $0.25 \mathrm{M} \mathrm{NaOH}(2.6 \mathrm{eq}$ ) was added. After $10 \mathrm{~min}$, a white precipitate formed, so more THF (4 ml) was added and the mixture was stirred at room temperature till the disappearance of the starting material monitored by TLC using EtOAc/hexanes 2:3 as eluent $(\sim 5 \mathrm{~h})$. After that, the mixture was acidified to $\mathrm{pH} 2$ with $1 \mathrm{M} \mathrm{HCl}$ and extracted with $\mathrm{CH}_{2} \mathrm{Cl}_{2}$. The organic phase was dried over $\mathrm{MgSO}_{4}$ and concentrated, under reduced pressure, to give a white solid that was crystallized from $\mathrm{CH}_{2} \mathrm{Cl}_{2} /$ pentane (vapor diffusion) to give $0.17 \mathrm{~g}$ (0.57 mmol, $98 \%$ yield) as a white crystalline solid. m.p. $114-116^{\circ} \mathrm{C}$ (from pentane). $[\alpha]_{\mathrm{D}}{ }^{20}+25.6(c 0.5$, $\left.\mathrm{CH}_{3} \mathrm{OH}\right)$. IR 3259.0, 2917.0, 2848.1, 1700.0, 1600.0, 1547.1 $\mathrm{cm}^{-1} .{ }^{1} \mathrm{H}$ NMR $\left(600 \mathrm{MHz}, \mathrm{CDCl}_{3}\right) \delta 0.87(\mathrm{t}$, $3 \mathrm{H}, J=6.8 \mathrm{~Hz}), 1.25$ (br m, 16H), 1.63 (br m, 2H), $1.90(\mathrm{~m}, 1 \mathrm{H}), 2.21-2.28(\mathrm{~m}, 5 \mathrm{H}), 3.13(\mathrm{~m}, 1 \mathrm{H}), 4.18$ (m, 1H), 6.47 (br s, 1H) ppm. ${ }^{13} \mathrm{C}$ NMR $\left(151 \mathrm{MHz}, \mathrm{CDCl}_{3}\right) \delta 14.2\left(\mathrm{CH}_{3}\right), 19.0,22.8,23.16,23.6,25.3-$ 29.7, 32.0, $36.0\left(\mathrm{CH}_{2}\right.$ chain and cyclobutane), $48.0(\mathrm{CH}), 49.0(\mathrm{CH}), 174.6(\mathrm{C}), 176.4(\mathrm{C}) \mathrm{ppm} . \mathrm{HRMS}$ (ESI+) Calcd. Mass for $\mathrm{C}_{17} \mathrm{H}_{31} \mathrm{NO}_{3} \mathrm{Na}[\mathrm{M}+\mathrm{Na}]^{+}:$320.2196. Found: 320.2207.

Sodium (1S,2S)-2-(dodecanamido)cyclobutanecarboxylate (trans-1). The sodium salt trans-1 was prepared according to the salification procedure reported above, starting from the intermediate acid trans$4(0.170 \mathrm{~g}, 0.57 \mathrm{mmol})$. The product obtained after lyophilization was washed with acetone and pentane affording $0.182 \mathrm{~g}$ (quantitative yield) of a white solid. m.p. $165-167{ }^{\circ} \mathrm{C}$ (from pentane). $[\alpha]_{\mathrm{D}}{ }^{20}+58.7(c$ 0.5, $\left.\mathrm{CH}_{3} \mathrm{OH}\right)$. IR $3305.3(\mathrm{NH}), 2918.3,2851.6\left(\mathrm{CH}\right.$ st), 1619.8 (amide I), $1563.7\left(\mathrm{COO}^{-}\right), 1545.5$ (amide II), $1421.4\left(\mathrm{COO}^{-}\right) \mathrm{cm}^{-1} .{ }^{1} \mathrm{H}$ NMR $\left(360 \mathrm{MHz}, \mathrm{CD}_{3} \mathrm{OD}\right) \delta 0.90(\mathrm{t}, 3 \mathrm{H}, J=6.5 \mathrm{~Hz}), 1.29$ (br m, 16H), 1.57 (br m, 2H), 1.65-2.00 (complex absorption, 3H), 2.10-2.22 (complex absorption, 3H), 2.89 (q, 1H, $J=9.1$ $\mathrm{Hz}), 4.37(\mathrm{q}, 1 \mathrm{H}, J=8.3 \mathrm{~Hz}) \mathrm{ppm} .{ }^{13} \mathrm{C} \mathrm{NMR}\left(90 \mathrm{MHz}, \mathrm{CD}_{3} \mathrm{OD}\right) \delta 14.4\left(\mathrm{CH}_{3}\right), 20.6,23.7,27.0,28.0$, 30.3-30.7, 33.0, $37.0\left(\mathrm{CH}_{2}\right.$ chain and cyclobutane), 49.4 ( $\mathrm{CH}$ overlapped to $\mathrm{CD}_{3} \mathrm{OD}$ signal), $50.2(\mathrm{CH})$, 175.2 (C), 181.8 (C) ppm. HRMS (ESI-) Calcd. Mass for $\mathrm{C}_{17} \mathrm{H}_{30} \mathrm{NO}_{3}{ }^{-}$[M-Na]: 296.2231. Found: 296.2224. 
${ }^{1} \mathrm{H}-\mathrm{NMR}\left(\mathrm{CDCl}_{3}, 360 \mathrm{MHz}\right)$ and ${ }^{13} \mathrm{C}-\mathrm{NMR}\left(\mathrm{CDCl}_{3}, 90 \mathrm{MHz}\right)$ of cis-3
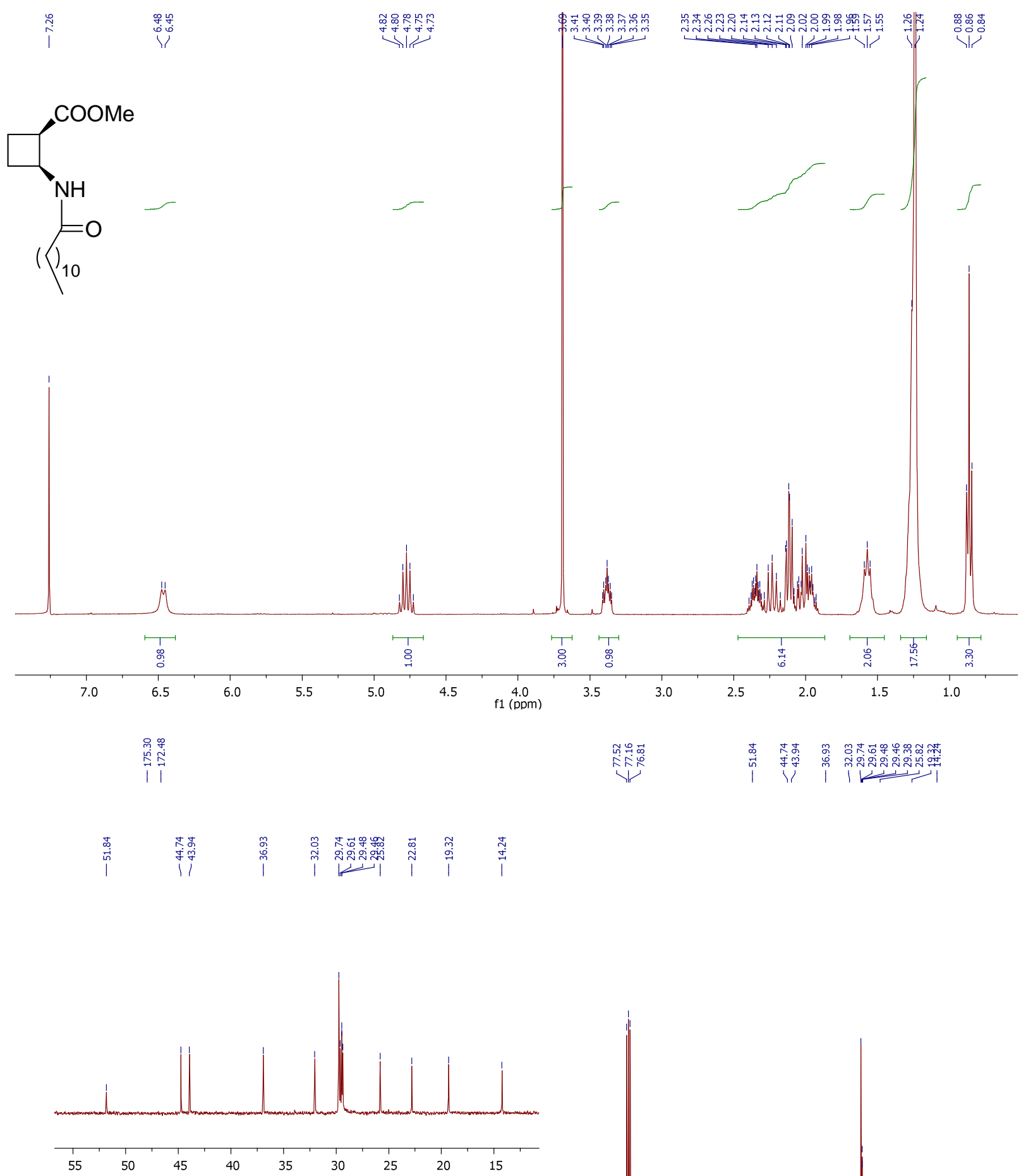


\section{${ }^{1} \mathrm{H}-\mathrm{NMR}\left(\mathrm{CDCl}_{3}, 250 \mathrm{MHz}\right)$ and ${ }^{13} \mathrm{C}-\mathrm{NMR}\left(\mathrm{CDCl}_{3}, 62.5 \mathrm{MHz}\right)$ of cis-4}

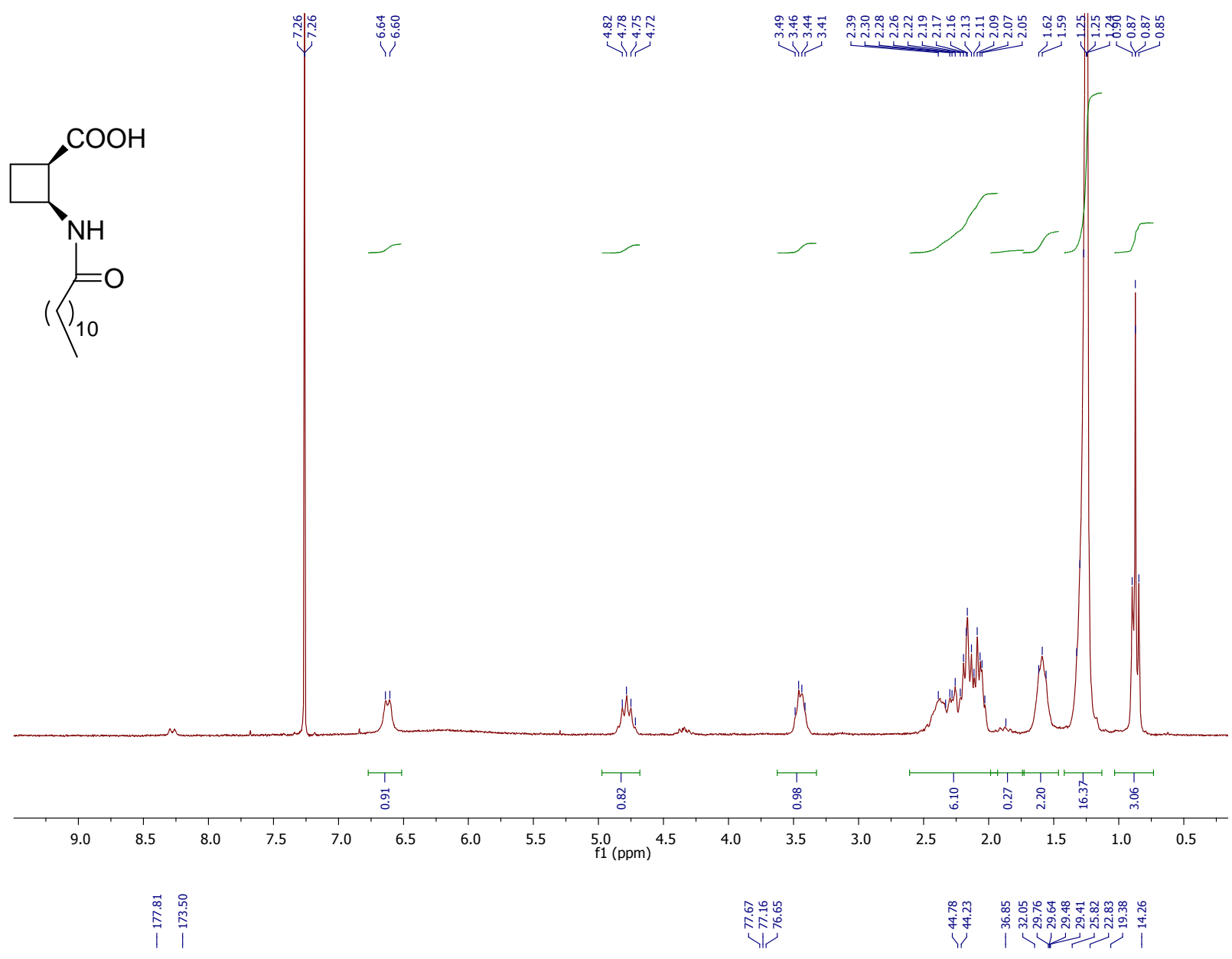

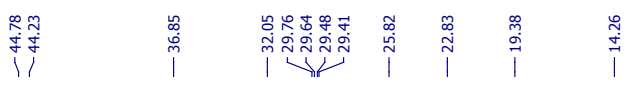
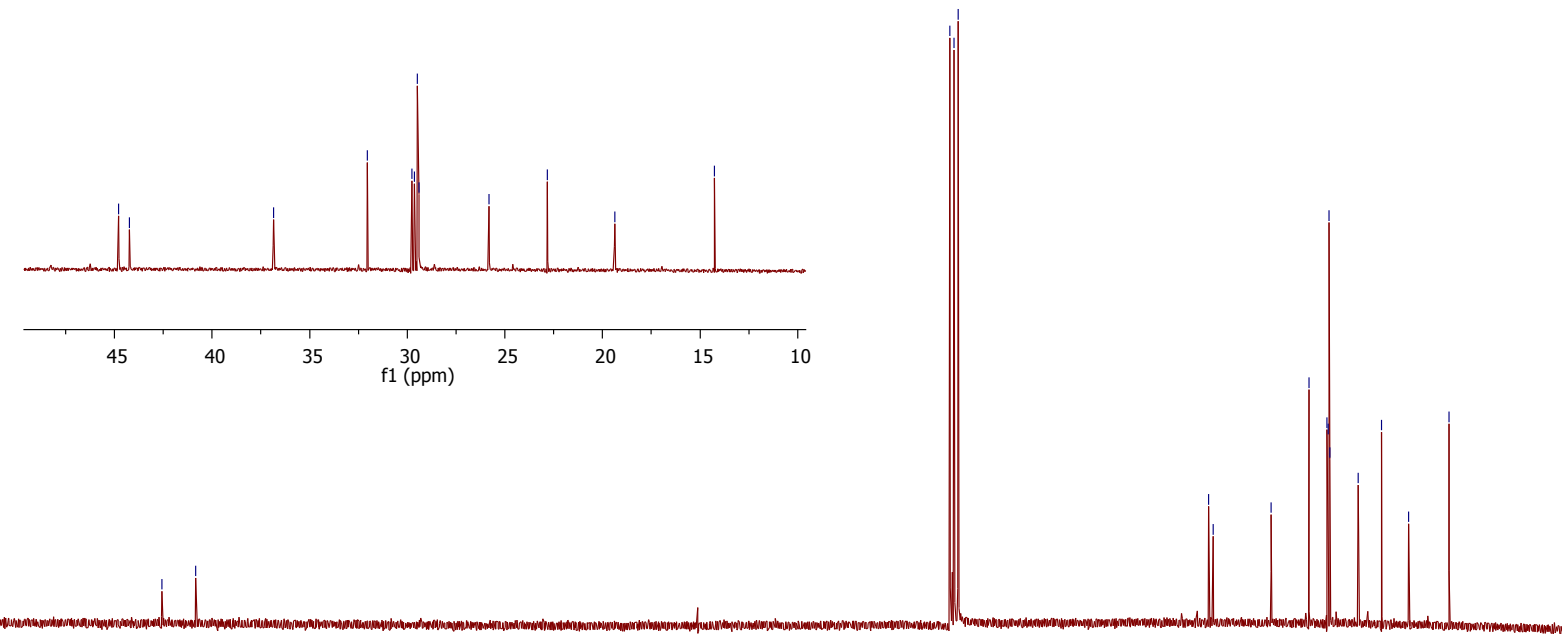
${ }^{1} \mathrm{H}-\mathrm{NMR}\left(\mathrm{CD}_{3} \mathrm{OD}, 360 \mathrm{MHz}\right)$ and ${ }^{13} \mathrm{C}-\mathrm{NMR}\left(\mathrm{CD}_{3} \mathrm{OD}, 90 \mathrm{MHz}\right)$ of $c$ is-1

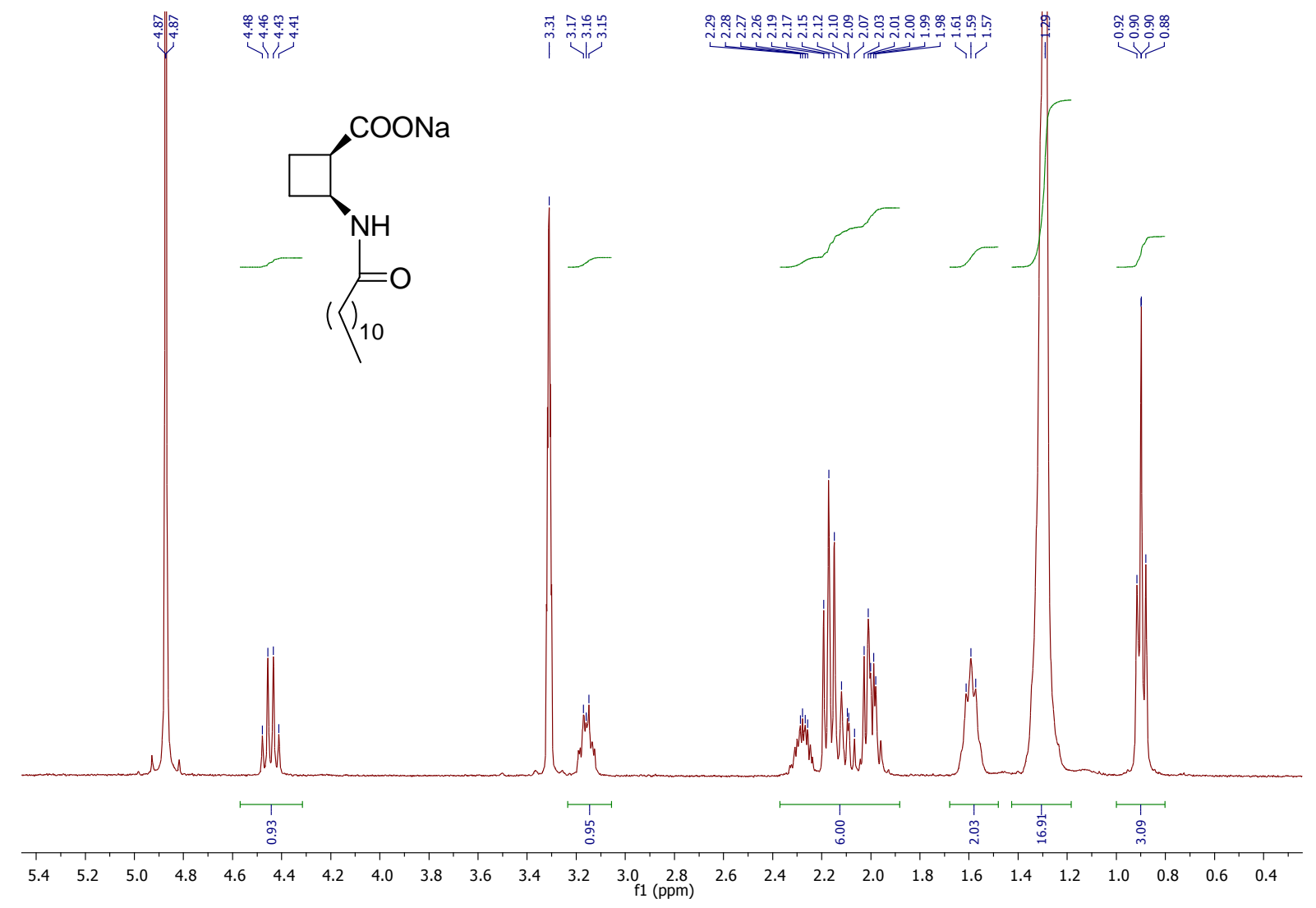

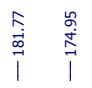

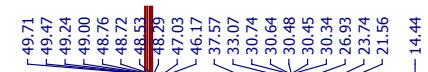
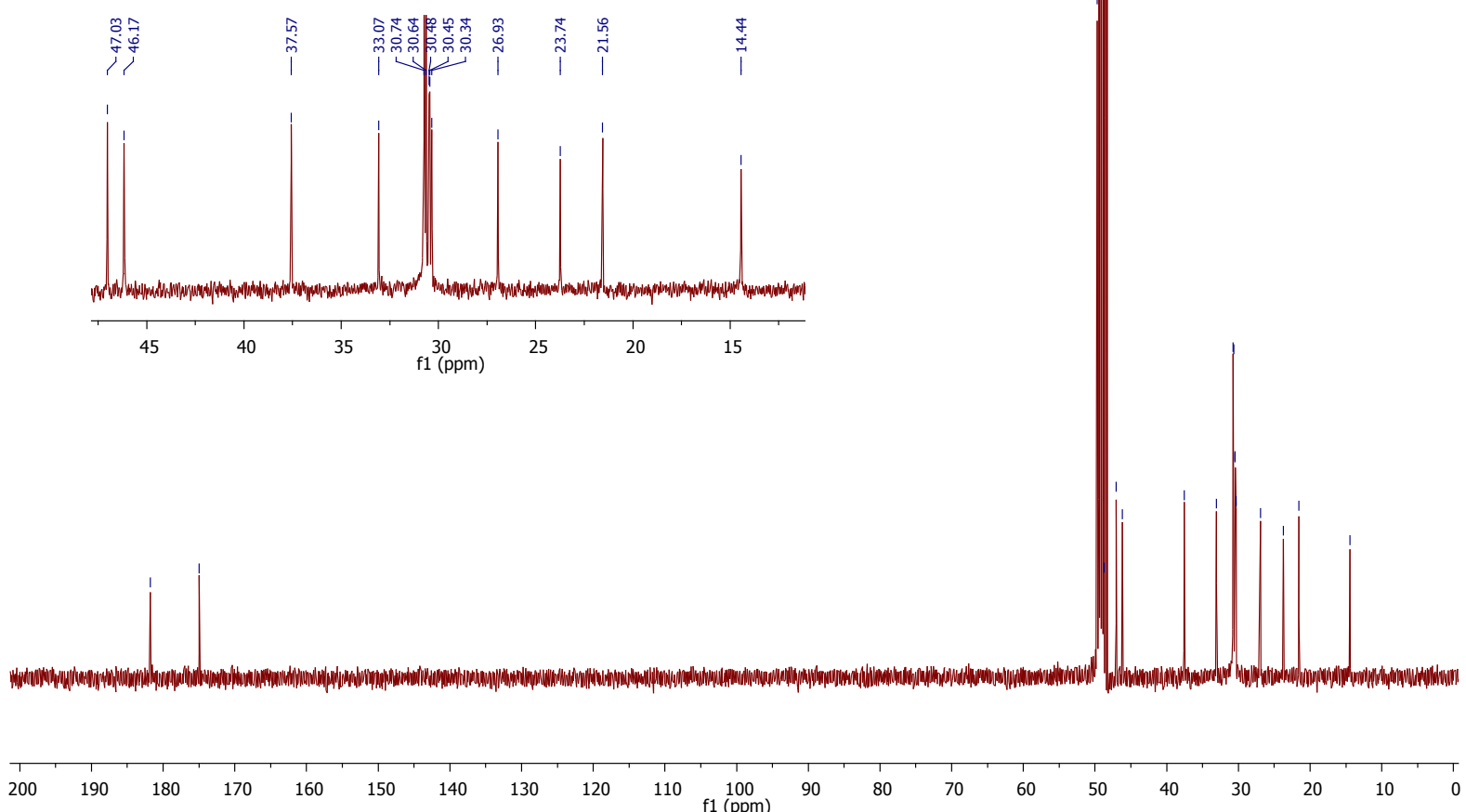
${ }^{1} \mathrm{H}-\mathrm{NMR}\left(\mathrm{CDCl}_{3}, 250 \mathrm{MHz}\right)$ and ${ }^{13} \mathrm{C}-\mathrm{NMR}\left(\mathrm{CDCl}_{3}, 90 \mathrm{MHz}\right)$ of trans-3

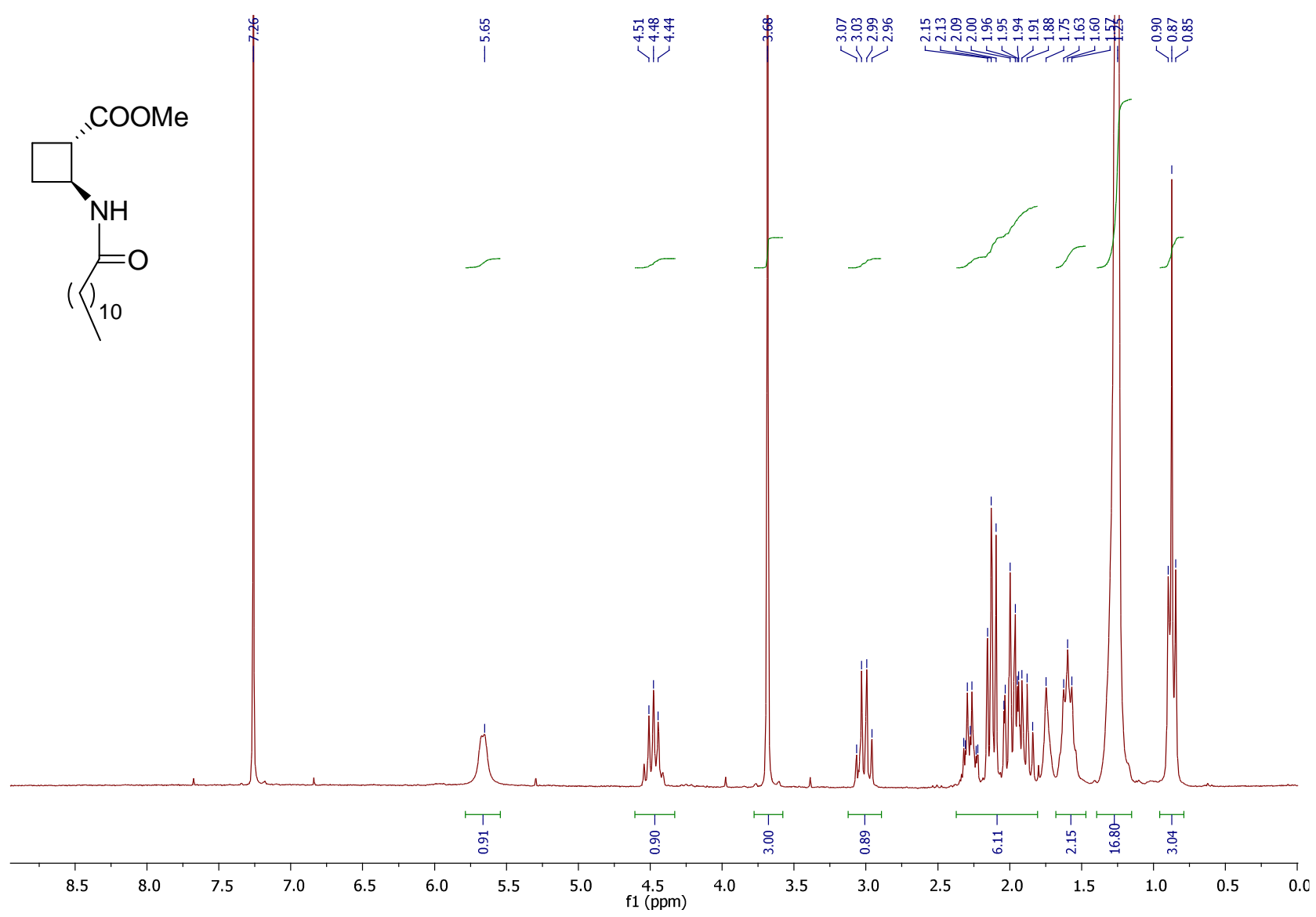

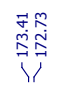

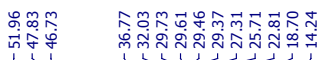

iर
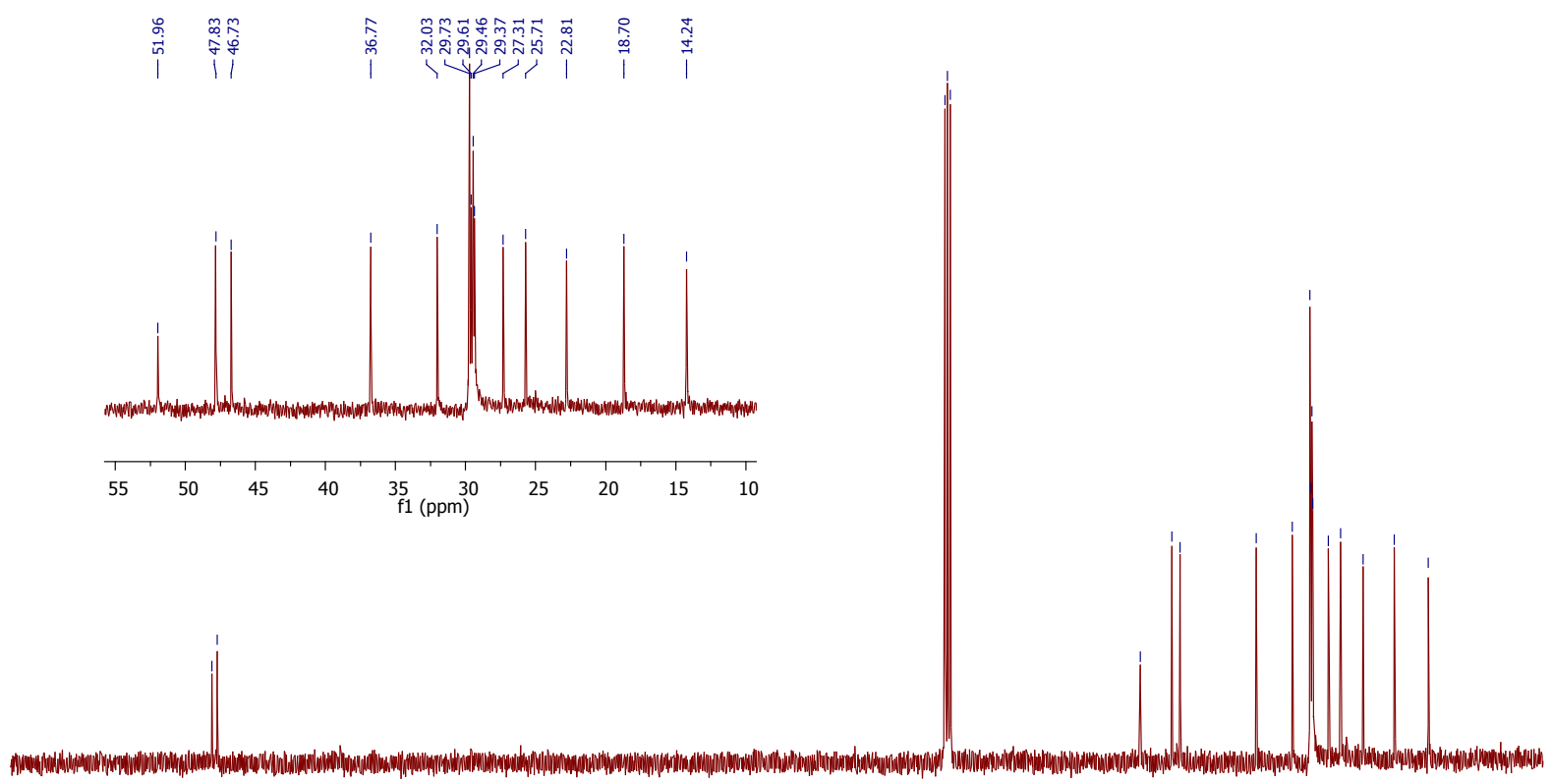

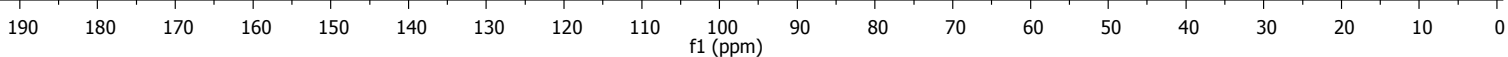

${ }^{1} \mathrm{H}-\mathrm{NMR}\left(\mathrm{CDCl}_{3}, 600 \mathrm{MHz}\right)$ and ${ }^{13} \mathrm{C}-\mathrm{NMR}\left(\mathrm{CDCl}_{3}, 151 \mathrm{MHz}\right)$ of trans-4 

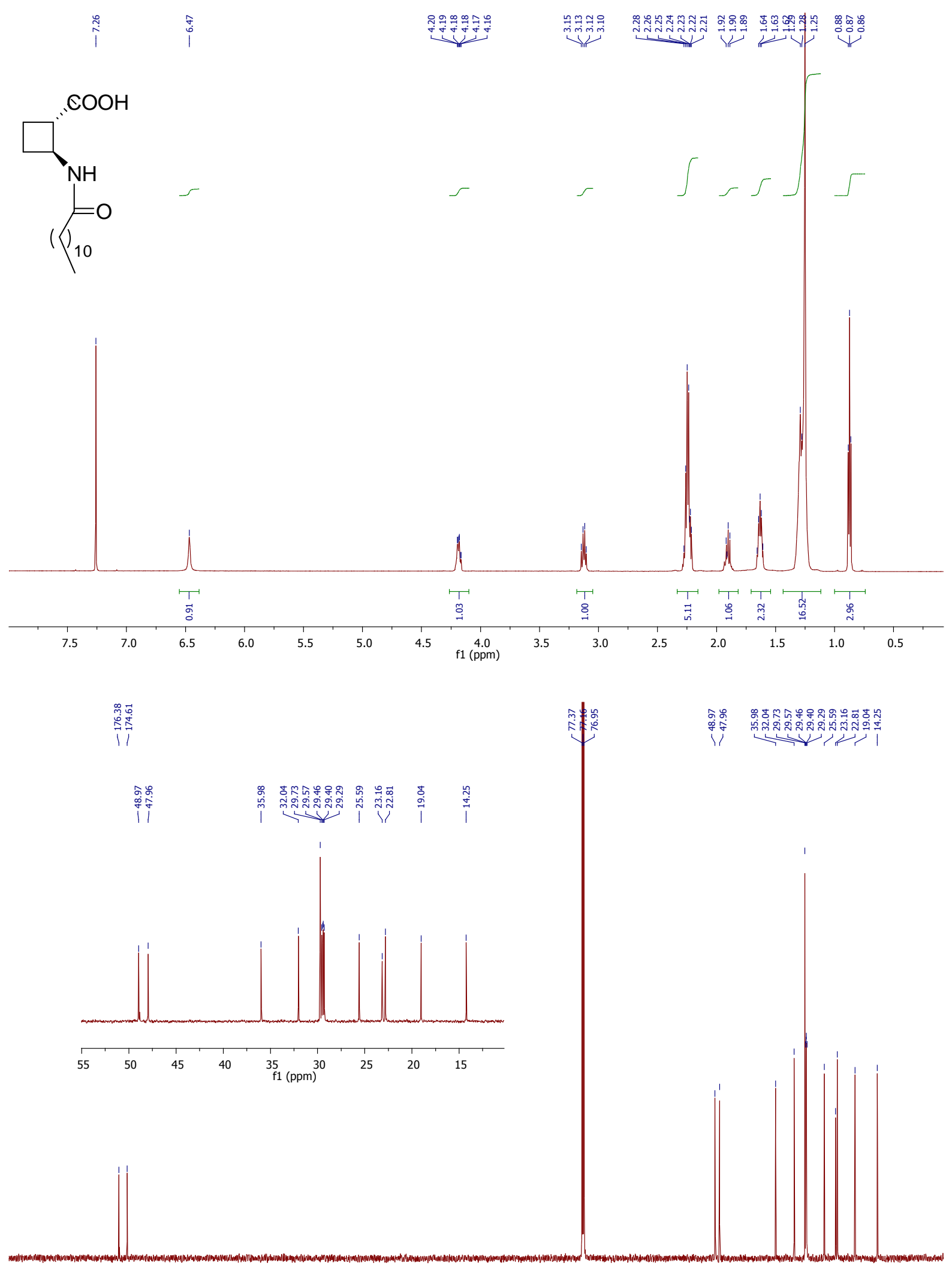

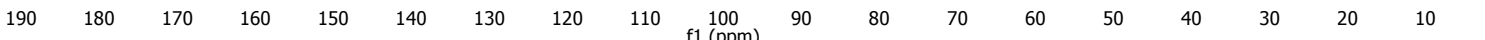

${ }^{1} \mathrm{H}-\mathrm{NMR}\left(\mathrm{CD}_{3} \mathrm{OD}, 360 \mathrm{MHz}\right)$ and ${ }^{13} \mathrm{C}-\mathrm{NMR}\left(\mathrm{CD}_{3} \mathrm{OD}, 90 \mathrm{MHz}\right)$ of trans-1 

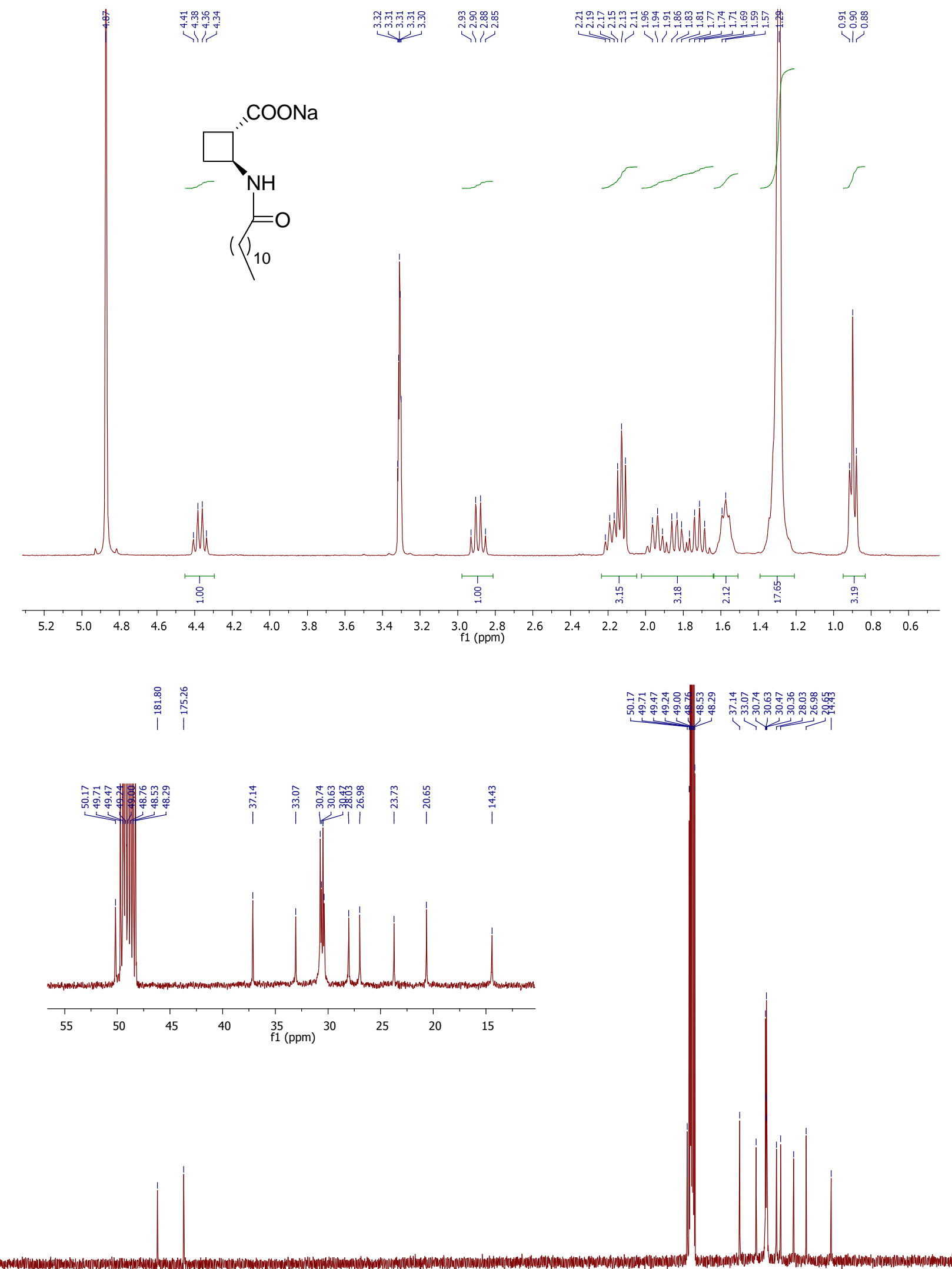

$\begin{array}{llllllllllllllllllllllllllllllllllll} & 220 & 210 & 200 & 190 & 180 & 170 & 160 & 150 & 140 & 130 & 120 & 110 & 100 & 90 & 80 & 70 & 60 & 50 & 40 & 30 & 20 & 10 & 0 & -10\end{array}$ 


\section{pH measurements:}

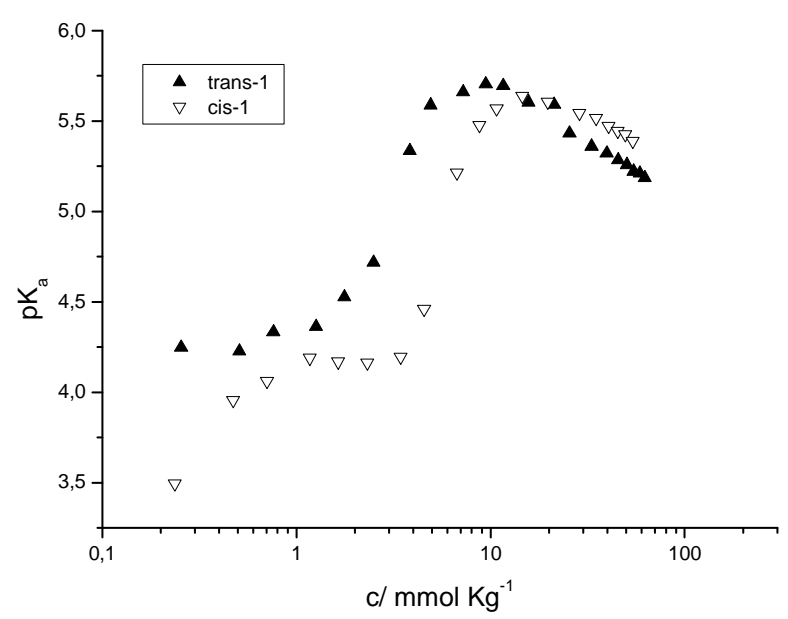

Figure S1: Apparent $\mathrm{pK}_{\mathrm{a}}$ as a function of surfactant concentration for the salts trans-1 and cis-1.

The $\mathrm{pK}_{\mathrm{a}}$ values both below the $c m c$ (surfactant as monomer) and above the $c m c$ (apparent $\mathrm{pK}_{\mathrm{a}}$ ) were evaluated by using the Equation 2 of the main text, that was developed as follow:

Equilibrium constant definition $K_{a}=\frac{\left[\mathrm{Ac}^{-}\right]\left[\mathrm{H}^{+}\right]}{[\mathrm{AcH}]}$

Mass balances $\mathrm{C}_{\mathrm{b}}=\left[\mathrm{Na}^{+}\right]$

$$
\mathrm{C}_{\mathrm{b}}=\left[\mathrm{Ac}^{-}\right]+[\mathrm{AcH}]
$$

Charge balance $\left[\mathrm{Ac}^{-}\right]+\left[\mathrm{OH}^{-}\right]=\left[\mathrm{H}^{+}\right]+\left[\mathrm{Na}^{+}\right]$

Water product $K_{w}=\left[\mathrm{H}^{+}\right]+\left[\mathrm{OH}^{-}\right]$

Using 5 in $4\left[\mathrm{Ac}^{-}\right]+K_{w} /\left[\mathrm{H}^{+}\right]=\left[\mathrm{H}^{+}\right]+\left[\mathrm{Na}^{+}\right]$

Using 2 in $4^{\prime}$ and adding $3 K_{w} /\left[\mathrm{H}^{+}\right]-\left[\mathrm{H}^{+}\right]=[\mathrm{AcH}]$

Using $2^{\prime}$ in $3 \mathrm{C}_{\mathrm{b}}=\left[\mathrm{Ac}^{-}\right]+K_{w} /\left[\mathrm{H}^{+}\right]-\left[\mathrm{H}^{+}\right]$

Using 1 in $3^{\prime} \mathrm{C}_{\mathrm{b}}=K_{a}[\mathrm{AcH}] /\left[\mathrm{H}^{+}\right]+\mathrm{K}_{\mathrm{w}} /\left[\mathrm{H}^{+}\right]-\left[\mathrm{H}^{+}\right]$

Using 2' in 1' leads to $\mathrm{C}_{\mathrm{b}}=K_{a}\left(K_{w} /\left[\mathrm{H}^{+}\right]-\left[\mathrm{H}^{+}\right]\right) /\left[\mathrm{H}^{+}\right]+K_{w} /\left[\mathrm{H}^{+}\right]-\left[\mathrm{H}^{+}\right]$ 
Which after some rearrangement can be put as:

$\mathrm{C}_{\mathrm{b}}=\left(K_{a}+\left[\mathrm{H}^{+}\right]\right) \cdot\left(K_{w} /\left[\mathrm{H}^{+}\right]^{2}-1\right)$

[Equation 2]

Where giving values to $\left[\mathrm{H}^{+}\right]$we can obtain $\mathrm{C}_{\mathrm{b}}$.

We can also isolate $\mathrm{K}_{\mathrm{a}}$ as a function of $\mathrm{C}_{\mathrm{b}}$ and $\left[\mathrm{H}^{+}\right]$to obtain values of apparent $\mathrm{Ka}$.

\section{$\underline{\text { SAXS measurement in diluted (micellar) solution of surfactants } 1}$}

Table S1. Partial volumes used in SAXS calculations

\begin{tabular}{|c|c|}
\hline Group & volume \\
\hline$-\mathrm{CH}_{3}$ & $0.055^{1}$ \\
\hline$-\mathrm{CH}_{2-}$ & $0.027^{1}$ \\
\hline$-\mathrm{C}_{4} \mathrm{H}_{6}-($ cyclobutane $)$ & $0.0904^{2}$ \\
\hline -COONa & $0.049^{1}$ \\
\hline$-\mathrm{COOH}$ & $0.045^{1}$ \\
\hline$-\mathrm{COOCH}_{3}$ & $\mathbf{0 . 0 8 5}^{1}$ \\
\hline -CONH- & $0.016^{3}$ \\
\hline
\end{tabular}

1 Pinazo, A.; Pérez, L.; Lozano, M.; Angelet, M.; Infante, M.R.; Vinardell, M.P.; Pons, R. J. Phys. Chem. B 2008, 112, 8578-8585.

2 Calculated from cyclobutane and ethylcyclobutane densities.

3 Barton, Allan F.M., CRC Handbood of solubility parameters and other cohesion parameters., CRC press, Boca Raton, Florida, 1991, p 161. 


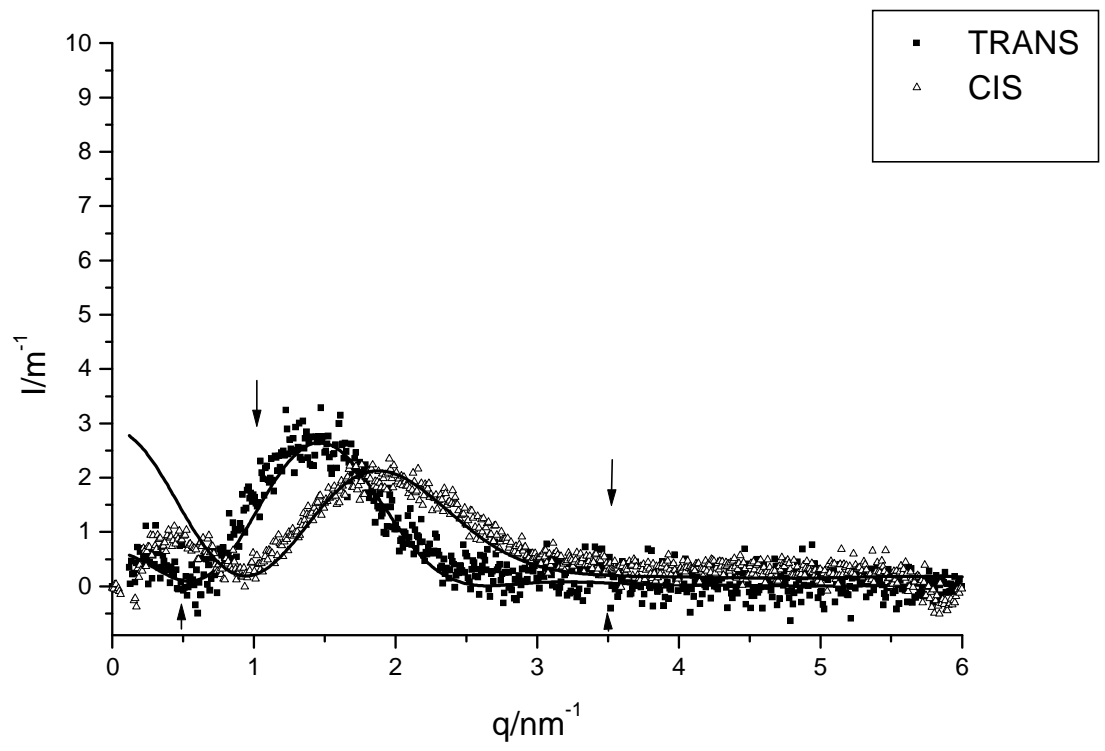

Figure S2. Intensity as a function of scattering vector modulus for freshly prepared trans-1 (full symbols) and cis1 (open symbols) samples. The full lines show the best fit of core-shell spheres. The up arrows show the fitting range for trans-1 while the down arrows show the fitting range for cis-1.

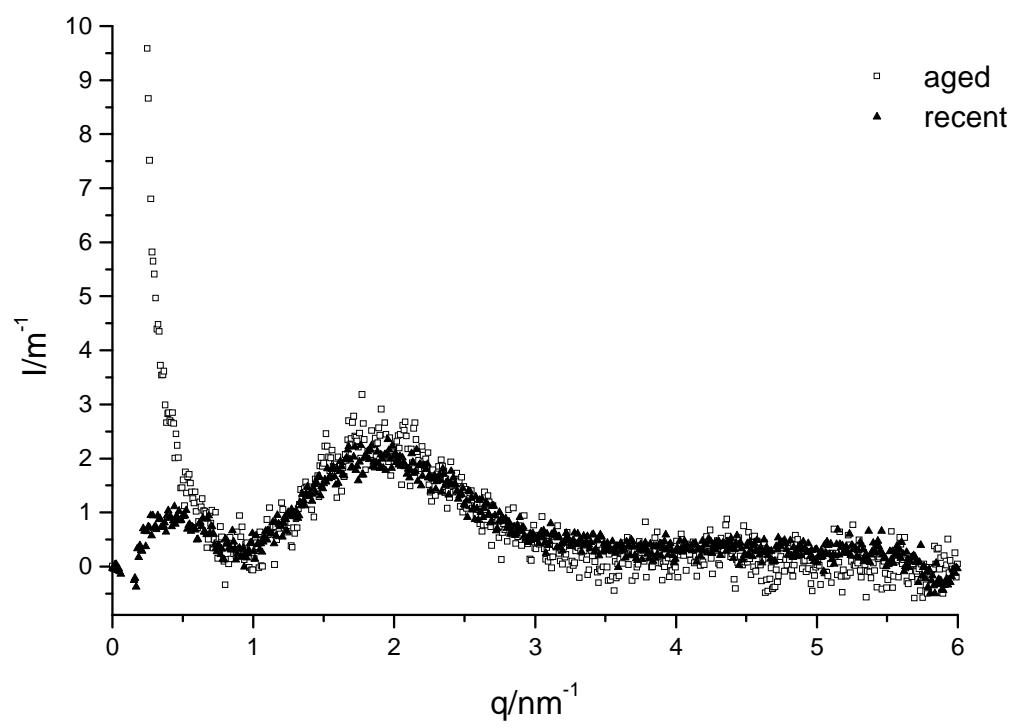

Figure S3. Intensity as a function of scattering vector modulus for freshly prepared cis-1 (full symbols) and $\underline{24 \mathrm{~h}}$ aged cis-1 (open symbols) samples. It is evident the increase of intensity at small q with time, that corresponds to the transition from spherical micelles to cylinders and ultimately to fibrils (see main text). 


\section{TEM and SEM images of fibrous assemblies formed by surfactant cis-1}
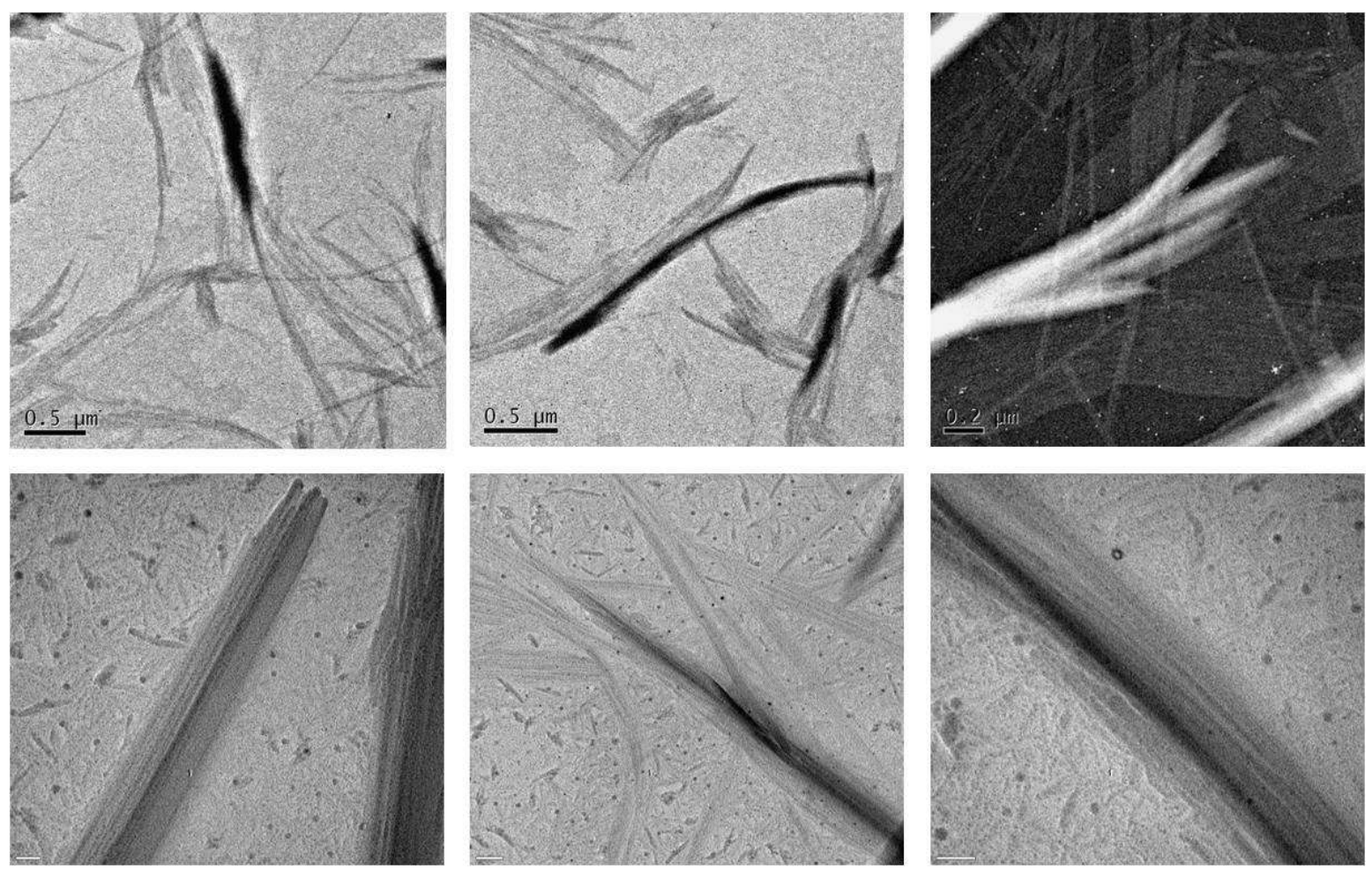

Figure S4. TEM images from a $24 \mathrm{~h}$ aged $1 \%$ water solutions of cis-1. The images of the upper row correspond to an unstained sample deposited on a carbon-coated copper grid: the images of the lower row were obtained after covering the sample with a thin Pt layer.
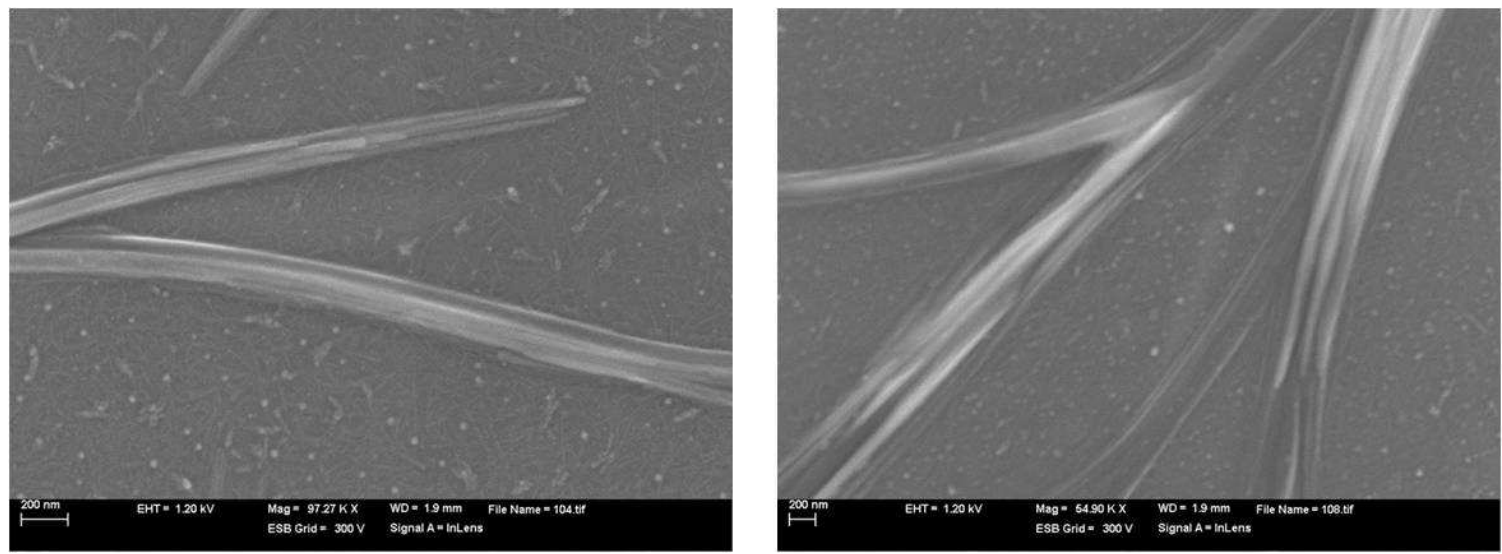

Figure S5. HR-SEM images of fiber formed by cis-1 surfactant (1\% in water) in a $24 \mathrm{~h}$ aged sample. The solution was deposited on a carbon-coated copper grid, the excess of water was blotted off and the sample was covered with a thin Pt layer. 

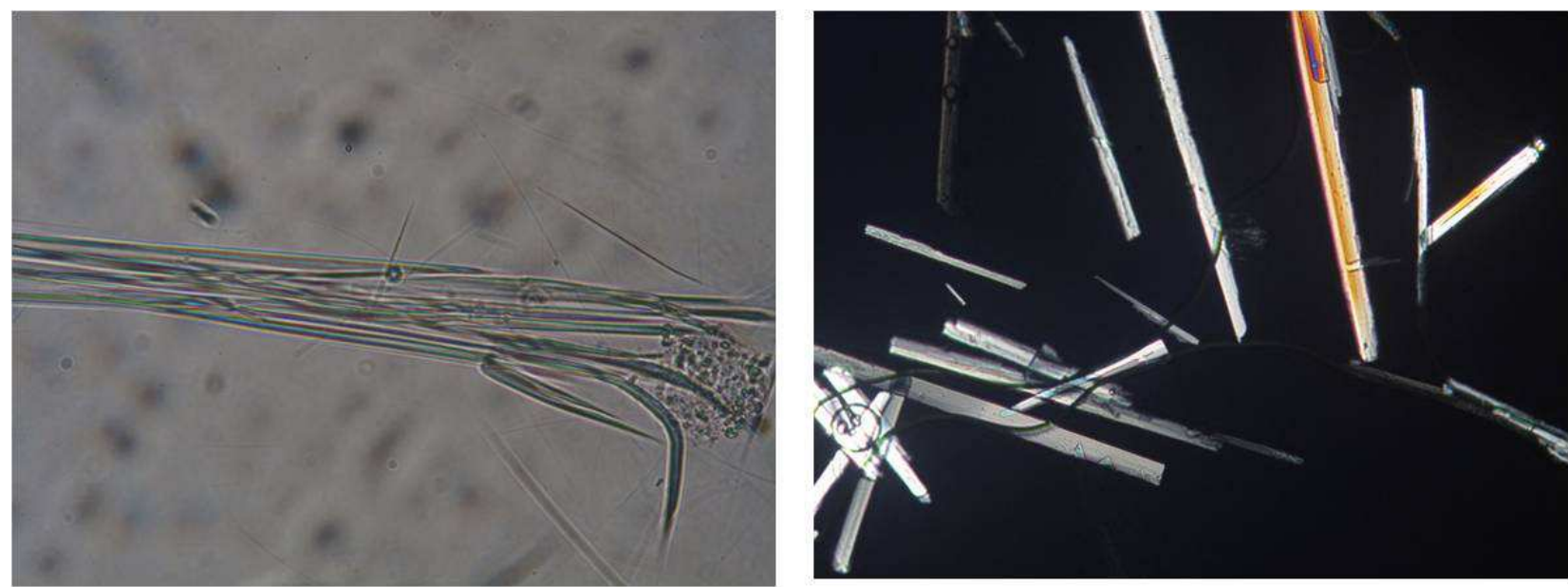

Figure S6. Electron micrographs of a drop of a 1 month aged 1\% water solutions of cis-1 (left, unpolarized; $211 \mathrm{x}$ $159 \mu \mathrm{m})$ and trans-1 (right, cross-polarized; $848 \times 636 \mu \mathrm{m}$ ) showing the different morphology of their fibrous aggregates.

\section{$\underline{\text { SAXS measurements of lyotropic liquid crystals of surfactants } 1}$}
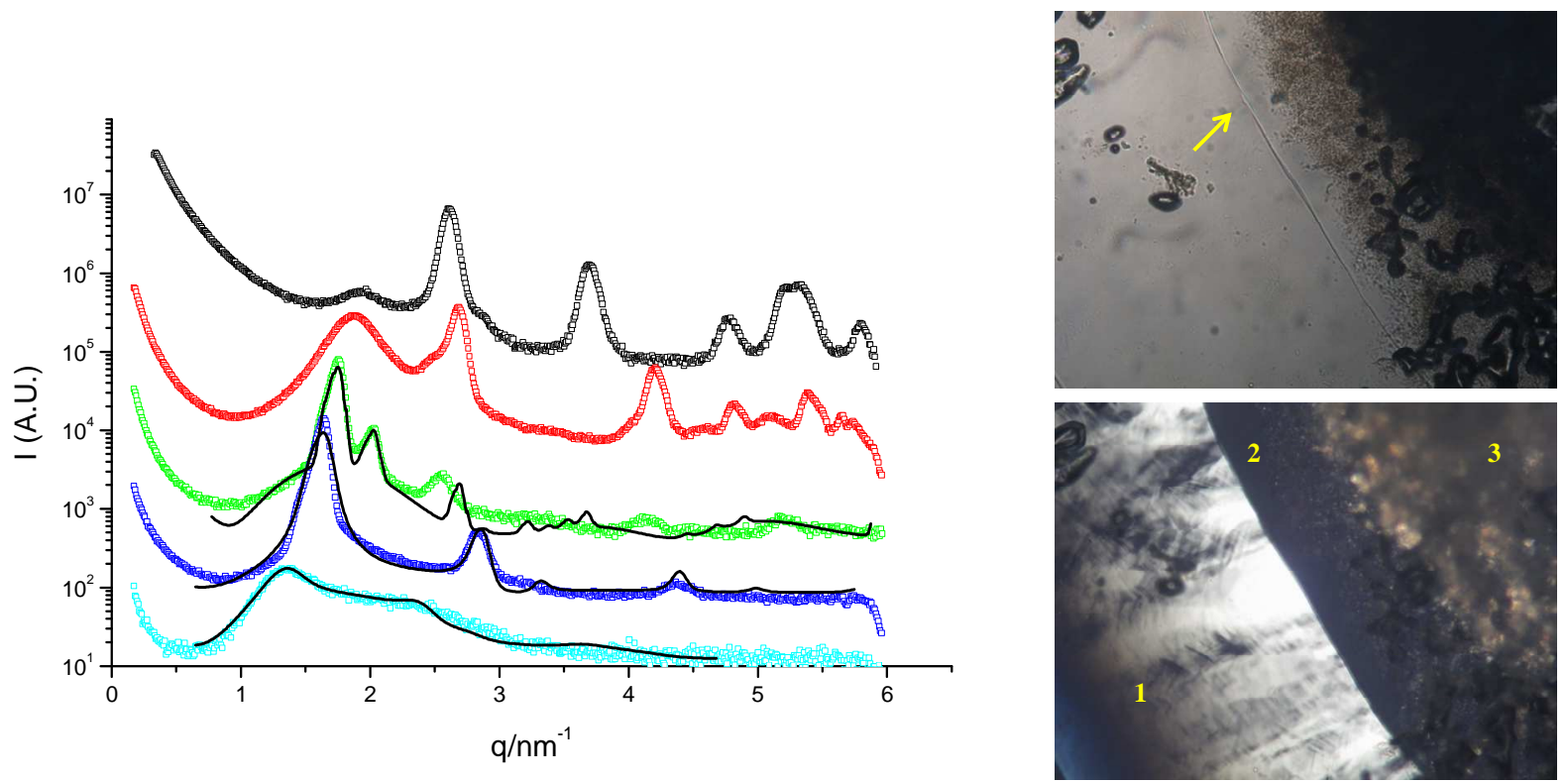

Figure S7. Left: SAXS profiles of $\boldsymbol{c i s - 1}$ mixtures with water. From bottom to top 30\%, 45\%, 60\%, and $80 \%$ w/w cis-1, and pure cis-1. The lines correspond to best fits to core-shell cylinders arranged in a hexagonal array for 30 and $45 \%$ and to Ia3d phase for the $60 \%$ sample. The samples were measured at $25^{\circ} \mathrm{C}$. Right: Non-polarized (top) and cross-polarized (bottom) optical micrographs $(848 \times 636 \mu \mathrm{m})$ relative to a water penetration experiment with cis-1. The patterns of region 1 are typical of a hexagonal phase while the dark fringe 2 and region 3 correspond respectively to the cubic phase and the dry solid. The arrow in the non-polarized image indicates the boundary between the hexagonal and the cubic phase. 


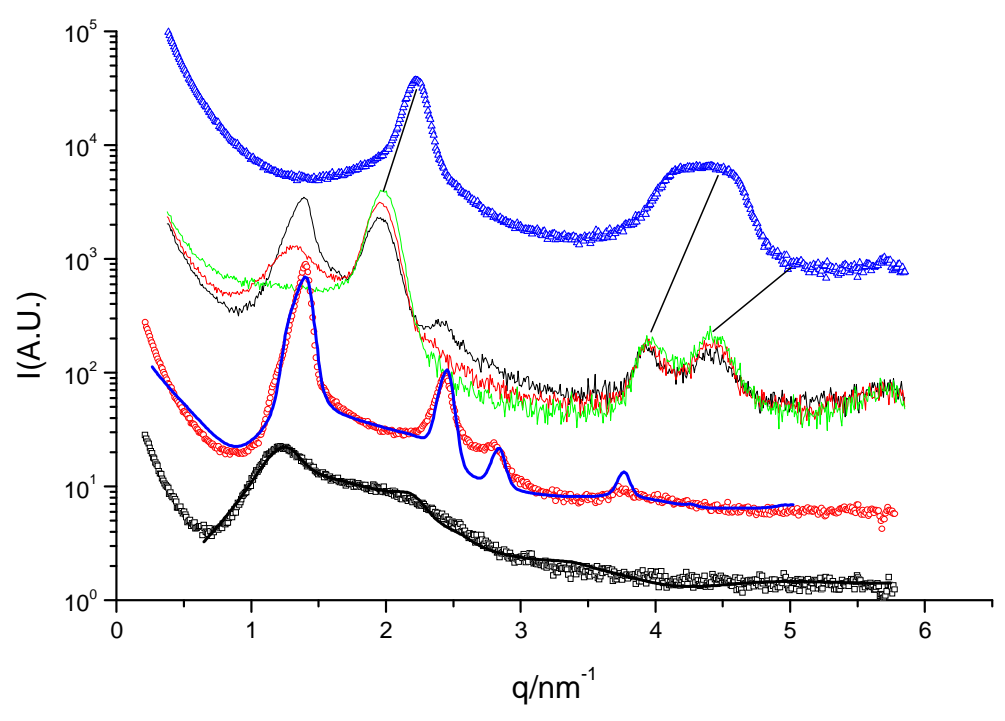

Figure S8. SAXS profiles of trans-1 mixtures with water. From bottom to top 30\%, 45\%, 60\% w/w trans-1 (at three successive times) and pure sample. The lines correspond to best fits to core-shell cylinders arranged in a hexagonal array. The samples were measured at $25^{\circ} \mathrm{C}$.

\section{Spectra of surfactants 1 and their aggregates}

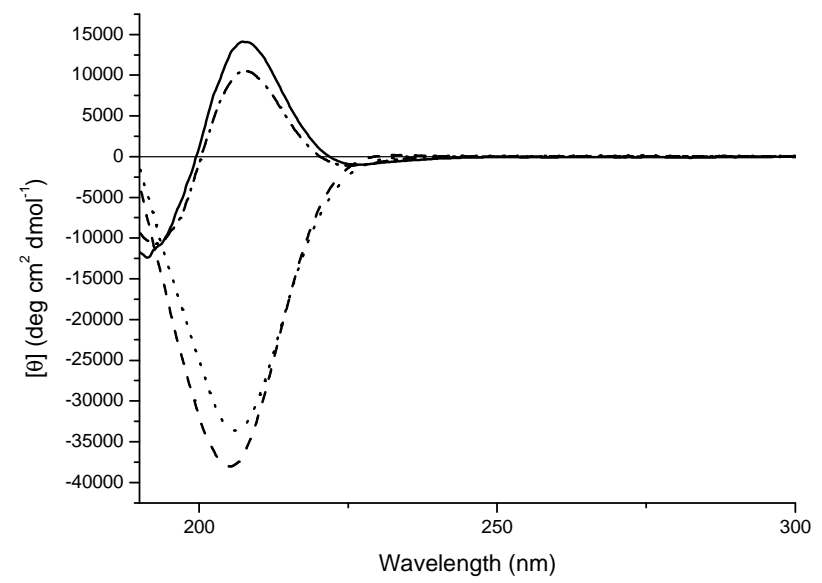

Figure S9. CD spectra of water solutions of cis-1: $2 \mathrm{mM}$ (dotted line) and $25 \mathrm{mM}$ (dashed line) and trans-1: $2 \mathrm{mM}$ (dash dotted line), $25 \mathrm{mM}$ trans-1 (solid line); optical path $0.01 \mathrm{~cm}$. 


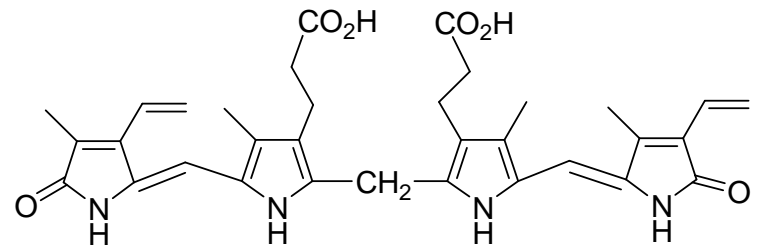

bilirubin-IX $\alpha$

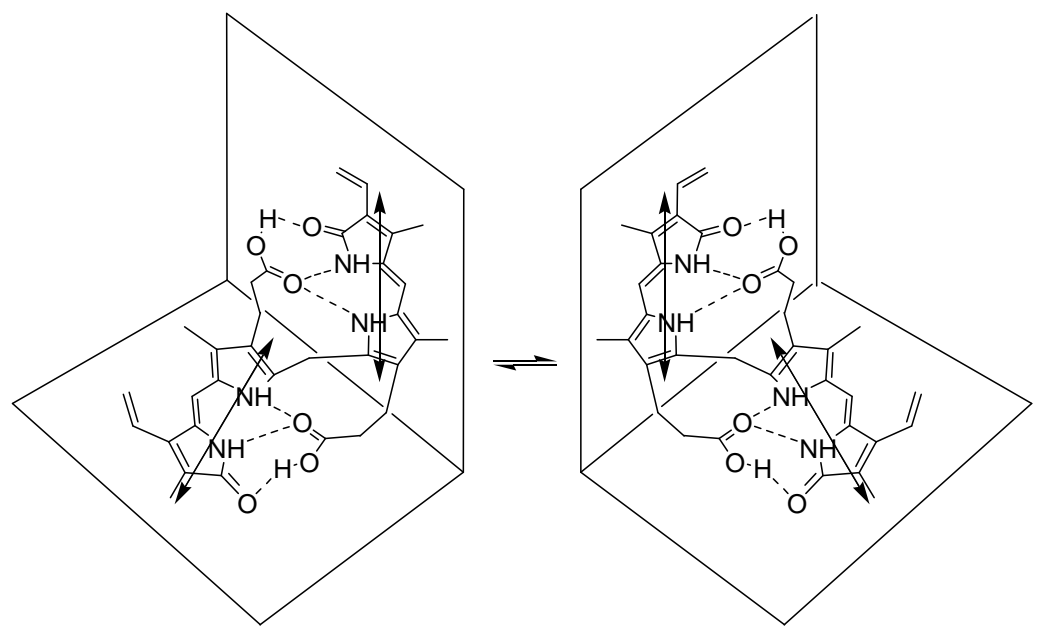

Scheme S1. Molecular structure and conformational enantiomers of bilirubin IX $\alpha$ 